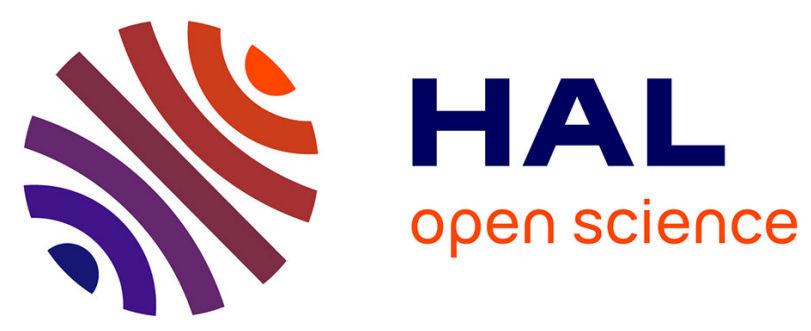

\title{
Size-dependent Eshelby tensor fields and effective conductivity of composites made of anisotropic phases with highly conducting imperfect interfaces
}

H. Le Quang, Guy Bonnet, Qi-Chang He

\section{- To cite this version:}

H. Le Quang, Guy Bonnet, Qi-Chang He. Size-dependent Eshelby tensor fields and effective conductivity of composites made of anisotropic phases with highly conducting imperfect interfaces. Physical Review B: Condensed Matter and Materials Physics (1998-2015), 2010, 81 (6), 10.1103/PhysRevB.81.064203 . hal-00692891

\section{HAL Id: hal-00692891 \\ https://hal.science/hal-00692891}

Submitted on 6 Mar 2016

HAL is a multi-disciplinary open access archive for the deposit and dissemination of scientific research documents, whether they are published or not. The documents may come from teaching and research institutions in France or abroad, or from public or private research centers.
L'archive ouverte pluridisciplinaire HAL, est destinée au dépôt et à la diffusion de documents scientifiques de niveau recherche, publiés ou non, émanant des établissements d'enseignement et de recherche français ou étrangers, des laboratoires publics ou privés. 


\title{
Size-dependent Eshelby tensor fields and effective conductivity of composites made of anisotropic phases with highly conducting imperfect interfaces
}

\author{
H. Le-Quang, * G. Bonnet, and Q.-C. He \\ Laboratoire Modelisation et Simulation Multi Echelle, Universite Paris-Est, MSME UMR 8208 CNRS, \\ 5 Boulevard Descartes, F-77454 Marne-la-Vallee Cedex 2, France
}

In this work, Eshelby's results and formalism for an elastic inhomogeneity embedded in an elastic infinite matrix are extended to the thermal-conduction phenomenon in composites consisting of anisotropic phases with highly conducting imperfect interfaces. The generalized Eshelby's interior and exterior conduction tensor fields and localization tensor fields in the important cases of circular and spherical inhomogeneities are obtained in an explicit analytical way. Quite different from the relevant results of elasticity, the generalized Eshelby's conduction tensor field and localization tensor field inside circular and spherical inhomogeneities are shown to remain uniform even in the presence of highly conducting imperfect interface. With the help of the obtained expressions for Eshelby's tensor fields and localization tensor fields, the size-dependent overall thermal-conduction properties of composites are estimated by using the dilute, Mori-Tanaka, self-consistent, and generalized self-consistent models. The analytical results are finally compared with numerical results delivered by the finite element method. The approach elaborated and results provided by the present work are directly applicable to other physically analogous transport phenomena, such as electric conduction, dielectrics, magnetism, diffusion, and flow in porous media and to the mathematically identical phenomenon of antiplane elasticity.

PACS number(s): 72.80.Tm, 05.60.Cd, 44.10.+i, 77.84.Lf

\section{INTRODUCTION}

In most of the studies dedicated to inhomogeneous materials, interfaces between constituent phases are assumed to be perfect. In the context of thermal conduction, an interface is called perfect if both the temperature and normal heat flux component are continuous across it. However, many composites of physical and engineering interest often exhibit imperfect contact between constituent phases. In studying the effects of imperfect interfaces on the effective conductivity of composites, use has mainly been made of the three models which, for the purpose of specifying the setting of the present work, are shortly described below.

The first model is based on Kapitza's concept of themal contact resistance which may result from the presence of material impurities or geometrical imperfections at phase boundaries. According to this model, the temperature suffers a jump across an interface while the normal heat flux component is continuous across the same interface and is usually taken to be proportional to the temperature jump. The effect of thermal resistance interfaces on the effective conductivity of composites has been widely investigated (see, e.g., Benveniste and Miloh, ${ }^{1}$ Benveniste, ${ }^{2}$ Hasselman and Johnson, ${ }^{3}$ Lipton and Vernescu, ${ }^{45}$ Torquato and Rintoul, ${ }^{6}$ Cheng and Torquato, ${ }^{7}$ Nan et al., ${ }^{8}$ and Hashin ${ }^{9}$ ).

The second model, referred to as the highly conducting (HC) interface model, can be considered as the limiting case associated with a very thin interphase of high conductivity located between two bulk media. According to this model, the temperature is continuous across an interface but the normal heat flux component is discontinuous across the same interface due to the possibility of having a surface heat flux along the interface. Investigation of the effect of highly conducting interfaces on the effective conductivity of composites has recently received particular attention (see, e.g.,
Torquato and Rintoul, ${ }^{6}$ Lipton, ${ }^{10,11}$ Miloh and Benveniste, ${ }^{12}$ and Yvonnet et $a l^{13}$ ).

The third model, referred to as the general imperfect interface model, is delivered by applying an asymptotic approach to a very thin interphase of uniform thickness situated between two bulk phases to obtain appropriate temperature and normal heat flux component jump conditions for an interface of zero thickness replacing the interphase (Hashin ${ }^{9}$ and Benveniste $\left.{ }^{14}\right)$. In this model, both the temperature and normal heat flux component are in general discontinuous across an interface. As shown early by Sanchez-Palencia ${ }^{15}$ and Pham Huy and Sanchez-Palencia, ${ }^{16}$ the general imperfect interface model reduces to the thermal resistance interface model or the highly conducting interface model according as the interphase is lowly conducting or highly conducting with respect to the surrounding phases. The governing equations of the general imperfect interface model involve not only the material parameters characterizing the interphase but also those relative to the surrounding phases. However, the governing equations of the thermal resistance interface model or the highly conducting interface model are independent of the material properties of the connected phases. The effect of the interfaces described by the general imperfect interface model on the effective conductivity of composites remains a largely open problem, even though a few particular cases were treated by Hashin $^{9}$ and Benveniste. ${ }^{14}$

The present work is concerned with the effective conductivity of composites consisting of a matrix in which circular inclusions [in the two-dimensional (2D) case] or spherical inclusions [in the three-dimensional (3D) case] are embedded via highly conducting interfaces. With respect to the works which have been done on this topic, the present one 
has two salient features: (i) the materials constituting the matrix and inclusions are not required to be isotropic but taken to have the most general thermal anisotropy, i.e. orthotropy and (ii) the effective conductivity of composites is estimated by preliminarily solving Eshelby's problem for a circular inclusion (in the two-dimensional case) or a spherical inclusion (in the three-dimensional case) inserted via an isotropic highly conducting interface into an infinite matrix. In contrast with the results presented by Sharma and Ganti ${ }^{17}$ and Duan et al. ${ }^{18}$ for Eshelby's problem with an interface being elastically equivalent to the highly conducting interface, our results show that the Eshelby's thermal-conduction tensor fields inside the circular or spherical inclusion are uniform. This uniformity makes it possible to transform the circular or spherical inhomogeneity problem into an equivalent circular or spherical inclusion problem and thus to preserve Eshelby's formalism even in the presence of a highly conducting interface. The temperature gradient and heat flux localization tensors can then be obtained and expressed in terms of Eshelby's tensors by applying the superposition principle. Finally, it is relatively easy to use the wellestablished micromechanical schemes to estimate the effective conductivity of composites under consideration.

The paper is organized as follows. Section II is dedicated to specifying the constitutive laws of the constituent phases of composites under investigation, the $\mathrm{HC}$ interface model and the general form of the effective thermal behavior. In Sec. III, the size-dependent Eshelby's interior and exterior tensor fields are determined in the context of conduction with $\mathrm{HC}$ imperfect interface. The size-dependent localization tensor fields are presented in Sec. IV. In Sec. V, closed-form expressions are derived for the effective conductivity moduli by using the dilute, Mori-Tanaka, self-consistent, and generalized self-consistent schemes. In Sec. VI, the HC interface and inhomogeneities size effects on the effective conductivity of composites are numerically discussed and illustrated; in addition, the closed-form solutions obtained are compared with the results provided by the finite element method (FEM). The paper is closed by drawing a few concluding comments in Sec. VII.

\section{LOCAL CONSTITUTIVE LAWS}

The composite under consideration consists of a matrix in which inclusions are embedded. Let $\Omega$ be the domain of a $2 \mathrm{D}$ or $3 \mathrm{D}$ Euclidean space $\mathcal{R}^{d}(d=2,3)$ occupied by a representative volume element of the composite and let $\partial \Omega$ be the boundary of $\Omega$. The subdomains of $\Omega$ inhabited by the inclusions and the matrix are denoted by $\Omega^{(1)}$ and $\Omega^{(2)}$, respectively. The interface between the matrix and inclusions is designed by $\Gamma$ [see Fig. 1(b)]. Relative to a right-handed orthonormal basis $\left\{\mathbf{f}_{i}\right\}(1 \leq i \leq d)$ in a system of Cartesian coordinates $\left\{x_{i}\right\}$, the matrix, referred to as phase 2 , and inclusions, denoted as phase 1 , are assumed to be individually homogeneous and have the linear thermal-conduction behavior described by an anisotropic Fourier's law

$$
\mathbf{q}^{(i)}=\mathbf{K}^{(i)} \mathbf{e}^{(i)} \text { or } \mathbf{e}^{(i)}=\mathbf{H}^{(i)} \mathbf{q}^{(i)} \text {. }
$$

Here $\mathbf{K}^{(i)}$ and $\mathbf{H}^{(i)}$, with $i=1$ or 2 , stand for the thermalconductivity and resistivity second-order tensors of phase $i$,

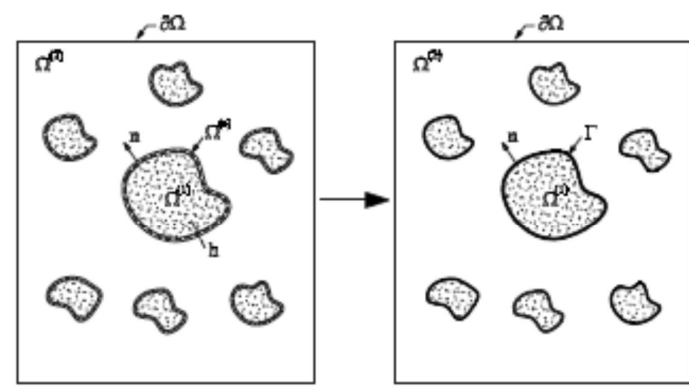

(8)

(b)

FIG. 1. Two- and three-phase configurations: (a) matrix/ interphase/inclusion composite and (b) matrix/inclusion composite with imperfect interface.

respectively, which are symmetric, positive definite and not necessarily isotropic. The intensity field $\mathrm{e}^{(i)}$ is related to the temperature field $T^{(i)}$ by

$$
\mathrm{e}^{(i)}=-\nabla T^{(i)}
$$

The heat flux field $q^{(i)}$ must verify the energy conservation equation

$$
\nabla \cdot \mathbf{q}^{(i)}=0
$$

in the case of stationary thermal conduction without heat source.

For later use, it is convenient to introduce the normal projection operator $\mathbf{P}^{\perp}$ and the tangent projection operator $\mathbf{P}$ of the interface $\Gamma$ between the matrix and inclusions defined by ${ }^{19}$

$$
\mathbf{P}^{\perp}(\mathbf{x})=\mathbf{n}(\mathbf{x}) \otimes \mathbf{n}(\mathbf{x}), \quad \mathbf{P}(\mathbf{x})=\mathbf{I}^{(d)}-\mathbf{n}(\mathbf{x}) \otimes \mathbf{n}(\mathbf{x}),
$$

where $\mathbf{n}(\mathbf{x})$ is the unit outward normal to $\Gamma$ and $\mathbf{I}^{(d)}$ stands for the $d$-dimensional second-order identity tensor. The interface $\Gamma$ between the matrix and inclusions is modeled by the $\mathrm{HC}$ interface model. According to the latter, the temperature field $T(\mathbf{x})$ and the tangential components of the intensity field $\mathbf{e}(\mathbf{x})$ are continuous across $\Gamma$, i.e., $\mathbf{P}(\mathbf{x}) \mathrm{e}^{(1)}(\mathbf{x})=\mathbf{P}(\mathbf{x}) \mathrm{e}^{(2)}(\mathbf{x})$ for any $\mathbf{x} \in \Gamma$. Indeed, the interface $\Gamma$ can be considered as a material surface and every point $\mathbf{x}$ of $\Gamma$ is endowed with a surface intensity field $\mathrm{e}^{s}(\mathbf{x})=\mathbf{P}(\mathbf{x}) \mathrm{e}^{(1)}(\mathbf{x})=\mathbf{P}(\mathbf{x}) \mathrm{e}^{(2)}(\mathbf{x})$ and a surface heat flux field $\mathbf{q}^{s}(\mathbf{x})$ which are related by the following surface Fourier isotropic law:

$$
\mathbf{q}^{s}(\mathbf{x})=\mathbf{K}_{s} \mathrm{e}^{s}(\mathbf{x}) \text { with } \mathbf{K}_{s}=k_{s} \mathbf{P}, \quad \mathbf{x} \in \Gamma .
$$

Here, $k_{s}$ stands for the surface thermal conductivity. Unlike the classical case where the interface is perfect, the normal component of the heat flux field $\mathbf{q}(\mathbf{x})$ is, in general, discontinuous across $\Gamma$ and its jump is related to the surface heat flux field $\mathbf{q}^{s}(\mathbf{x})$ by the following surface energy conservation equation:

$$
\left(\mathbf{q}^{(2)}-\mathbf{q}^{(1)}\right) \cdot \mathbf{n}(\mathbf{x})=-\operatorname{div}_{s} \mathbf{q}^{s}(\mathbf{x}), \quad \mathbf{x} \in \Gamma,
$$

where $\operatorname{div}_{s} \mathbf{q}^{s}(\mathbf{x})$ represents the surface divergence of $\mathbf{q}^{s}(\mathbf{x})$. In particular, $\operatorname{div}_{s} \mathbf{q}^{s}(\mathbf{x})$ takes the form (see, e.g., Chen et al. ${ }^{20}$ ) 


$$
\operatorname{div}_{s} \mathbf{q}^{s}(\mathbf{x})=\frac{\partial q_{\theta}^{s}}{r \partial \theta}+\frac{q_{\theta}^{s}}{r} \cot \theta+\frac{1}{r \sin \theta} \frac{\partial q_{\varphi}^{s}}{\partial \varphi}
$$

in the spherical coordinate system $(r, \theta, \varphi)$ or

$$
\operatorname{div}_{s} \mathbf{q}^{s}(\mathbf{x})=\frac{\partial q_{\theta}^{s}}{r \partial \theta}
$$

in the polar coordinate system $(r, \theta)$.

Next, in order to clarify the physical background and the validity domain of the $\mathrm{HC}$ interface model used to describe $\Gamma$, we consider in Fig. 1 two configurations. In the threephase one [Fig. 1(a)], a representative volume element $\Omega$ consists of the inclusions phase $\Omega^{(1)}$ embedded in the matrix phase $\Omega^{(2)}$ via the interphase $\Omega^{(s)}$. According to the interphase model, the interface between $\Omega^{(1)}$ and $\Omega^{(s)}$ and the interface between $\Omega^{(2)}$ and $\Omega^{(s)}$ are assumed to be perfect, and the thickness $h$ of $\Omega^{(s)}$ is required to be uniform and very small in comparison with the minimum size of inclusions. In the second configuration [Fig. 1(b)], $\Omega^{(s)}$ is now replaced by an interphase of zero thickness located at the middle surface $\Gamma$ of the interphase, and the inclusions and matrix are extended up to the middle surface $\Gamma$. By using asymptotic expansions, the jump conditions that the imperfect interface $\Gamma$ has to verify for the two configurations to be physically equivalent were derived first by Sanchez-Palencia ${ }^{15}$ and Pham Huy and Sanchez-Palencia ${ }^{16}$ in a particular case and then completed by Miloh and Benveniste, ${ }^{12}$ Hashin, ${ }^{9}$ and Benveniste ${ }^{14}$ in the general situation. More precisely, when the interphase with thermal-conductivity tensor $\mathbf{K}^{(\text {inser) }}$ is assumed to be highly conducting, or equivalently $\left\|\mathbf{K}^{(\text {inter })}\right\|$ $\geqslant\left\|\mathbf{K}^{(1)}\right\|$ and $\left\|\mathbf{K}^{(\text {inter })}\right\| \geqslant\left\|\mathbf{K}^{(2)}\right\|$, then the conditions that the imperfect interface $\Gamma$ must satisfy are those of the $\mathrm{HC}$ interface model described above. The corresponding surface thermal-conductivity tensor $\mathbf{K}_{s}$ of $\Gamma$ can be expressed in terms of $\mathbf{K}^{(\text {inter) }}$ and $h$ as follows (see Benveniste ${ }^{14}$ ):

$$
\mathbf{K}_{s}=h \mathbf{S}^{(s)},
$$

where

$$
\mathbf{S}^{(s)}=\mathbf{K}^{(\text {inter })}-\frac{\left(\mathbf{K}^{(\text {inter })} \mathbf{n}\right) \otimes\left(\mathbf{K}^{(\text {inter })} \mathbf{n}\right)}{\mathbf{K}^{(\text {inter })}:(\mathbf{n} \otimes \mathbf{n})} .
$$

In the present work, $\mathbf{K}^{(i n t e r)}$ is assumed to be isotropic, i.e., $\mathbf{K}^{(\text {inter })}=k_{\text {inter }} \mathbf{I}^{(d)}$ with $k_{\text {inter }}$ standing for the thermal conductivity of the interphase and it is immediate from Eqs. (9) and (10) that

$$
\mathbf{K}_{s}=k_{s} \mathbf{P}, \quad k_{s}=h k_{\text {inter }}
$$

Finally, at the macroscopic scale, the composite under consideration is assumed to be statistically homogeneous. The corresponding effective thermal behavior is characterized by

$$
\mathbf{Q}=\mathbf{K}^{e f f} \mathbf{E},
$$

where $\mathbf{K}^{e f f}$ is the effective thermal-conductivity secondorder tensor, $\mathbf{Q}$ and $\mathbf{E}$ denote the macroscopic heat flux and intensity fields, respectively. More precisely, the macroscopic intensity field $\mathbf{E}$ and heat flux field $\mathbf{Q}$ are defined as

$$
\begin{gathered}
\mathbf{E}=-\frac{1}{|\Omega|} \int_{\partial \Omega} T(\mathbf{x}) \nu(\mathbf{x}) d \mathbf{x}, \\
\mathbf{Q}=\frac{1}{|\Omega|} \int_{\partial \Omega}(\mathbf{q} \cdot \boldsymbol{v}) \mathbf{x} d \mathbf{x},
\end{gathered}
$$

where $\boldsymbol{\nu}(\mathbf{x})$ is the outward unit normal vector to $\alpha \Omega$ and $|\Omega|$ denotes the volume or surface of the domain $\Omega$ according as the $3 \mathrm{D}$ or $2 \mathrm{D}$ case is concerned. As for the classical case with perfect interfaces, the macroscopic intensity field $\mathbf{E}$ corresponds therefore to the volume or surface average of the local counterpart $\mathbf{e}(\mathbf{x})$ over $\Omega$, i.e.,

$$
\mathbf{E}=\frac{1}{|\Omega|} \int_{\Omega} \mathbf{e}(\mathbf{x}) d \mathbf{x}=\langle\mathbf{e}\rangle,
$$

where $\langle\boldsymbol{Q}\rangle$ is the volume or surface average of quantity over the domain $\boldsymbol{\Omega}$. However, unlike the classical case where the matrix-inclusion interface is perfect, the macroscopic heat flux field $Q$ is not simply the volume or surface average of the local counterpart $q(\mathbf{x})$ over $\Omega$ and it can be determined by

$$
\mathbf{Q}=\langle\mathbf{q}\rangle+\frac{1}{|\Omega|} \int_{\Gamma}\left(\mathbf{q}^{(2)} \cdot \mathbf{n}-\mathbf{q}^{(1)} \cdot \mathbf{n}\right) \mathbf{x} d \mathbf{x}
$$

or equivalently

$$
\mathbf{Q}=\langle\mathbf{q}\rangle-\frac{1}{|\Omega|} \int_{\Gamma}\left(\operatorname{div}_{s} \mathbf{q}^{s}\right) \mathbf{x} d \mathbf{x} .
$$

Thus, compared with the classical case, a new term due to the discontinuity of the normal component of the heat flux field across the HC interface $\Gamma$ appears in Eqs. (16) and (17).

\section{SIZE-DEPENDENT ESHELBY'S CONDUCTION TENSOR FIELDS}

In this section, we consider a $2 \mathrm{D}$ or $3 \mathrm{D}$ infinitely extended domain $\Omega$ made of a homogeneous material whose thermal conductivity and resistivity tensors are denoted by $\mathbf{K}^{0}$ and $\mathbf{H}^{0}$, respectively. Now, we are interested in the thermal counterpart of the well-known Eshelby's elastic problem ${ }^{21}$ while accounting for imperfect interface effects. Let a heat-free intensity $\mathbf{e}^{0}$, which plays a role similar to the eigenstrain in elasticity, be uniformly distributed in a subdomain $\omega$ of $\Omega$ and vanish outside $\omega$ (Fig. 2). Introducing the characteristic function $\chi^{\omega}(\mathbf{x})$ of $\omega$ by

$$
\chi^{\omega}(\mathbf{x})= \begin{cases}1 & \text { for } \mathbf{x} \in \omega, \\ 0 & \text { for } \mathbf{x} \notin \omega,\end{cases}
$$

the prescribed heat-free intensity field $\mathbf{e}^{*}(\mathbf{x})$ can be expressed as

$$
\mathbf{e}^{*}(x)=\chi^{\omega}(x) \mathbf{e}^{0} .
$$

The temperature field produced by $\mathbf{e}^{*}(\mathbf{x})$ is denoted by $T(\mathbf{x})$. The corresponding heat flux field $\mathbf{q}(\mathbf{x})$ is given by

$$
q(x)=K^{0} e(x)+q^{*}(x)
$$




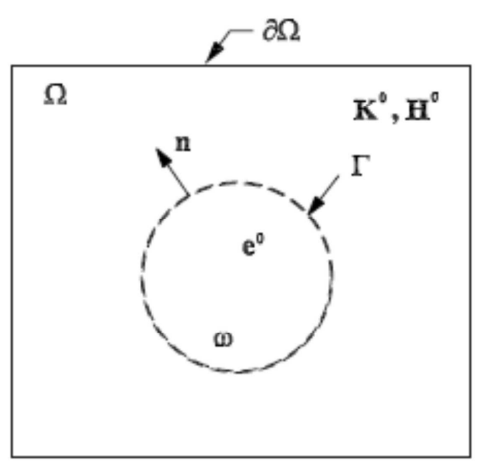

FIG. 2. The Eshelby's conduction problem consisting of a subdomain $\omega$ embedded in an infinite medium $\Omega$ and subjected to a uniform heat-free intensity $\mathrm{e}^{0}$ within $\omega$.

$$
q^{*}(x)=-K^{0} e^{*}(x)=-\chi^{\omega}(x) K^{0} e^{0} .
$$

Substituting Eq. (20) into Eq. (3) and accounting for the discontinuity of $\mathbf{q}(\mathbf{x})$ across interface $\Gamma$ yield

$$
\nabla \cdot \mathbf{q}(\mathbf{x})=\nabla \cdot\left[\mathbf{K}^{0} \mathbf{e}(\mathbf{x})\right]+p(\mathbf{x})-\left[\mathbf{q}^{(2)}-\mathbf{q}^{(1)}\right] \cdot \mathbf{n} \delta_{\Gamma}(\mathbf{x})=0,
$$

where

$$
p(\mathbf{x})=\nabla \cdot \mathbf{q}^{*}(\mathbf{x})
$$

and $\delta_{\mathrm{T}}$ is the Dirac delta function defined on $\Gamma$

By defining the Green's function for themal conduction, denoted by $\hat{G}(\mathbf{x}, \mathbf{y})$, which gives the temperature at point $\mathbf{x}$ produced by a unit point source applied at point $\mathbf{y}$, and by accounting for Eq. (6), the temperature field can be expressed in terms of $\hat{G}(\mathbf{x}, \mathbf{y})$ and $\mathbf{q}^{s}(\mathbf{y})$ as

$$
T(\mathbf{x})=\int_{\Gamma} \hat{G}(\mathbf{x}, \mathbf{y}) \operatorname{div}_{s} \mathbf{q}^{s}(\mathbf{y}) d \mathbf{y}+\int_{\Omega} \hat{G}(\mathbf{x}, \mathbf{y}) p(\mathbf{y}) d \mathbf{y} .
$$

Since the domain $\Omega$ under consideration is unbounded, the Green's function $\hat{G}(\mathbf{x}, \mathbf{y})$ can be expressed as a function of $\mathbf{y}-\mathbf{x}$ and denoted by $G(\mathbf{y}-\mathbf{x})$. For a domain containing a constant conduction tensor of components $K_{i j}^{0}, G(\mathbf{y}-\mathbf{x})$ is solution to the equation

$$
K_{i j}^{0} G_{i j}(\mathbf{y}-\mathbf{x})=\delta(\mathbf{y}-\mathbf{x}),
$$

where the subscript $i$ following a comma denotes the derivative with respect to $y_{i}$.

Taking into account that $\hat{G}(\mathbf{x}, \mathbf{y})=O\left(\|\mathbf{y}-\mathbf{x}\|^{-1}\right)$, the divergence theorem can be used to transform the second integral of Eq. (24), leading to

$$
\begin{aligned}
T(\mathbf{x})= & \int_{\Gamma} G(\mathbf{y}-\mathbf{x}) \operatorname{div}_{s} \mathbf{q}^{s}(\mathbf{y}) d \mathbf{y}-\int_{\Omega} \nabla G(\mathbf{y}-\mathbf{x}) \cdot \mathbf{q}^{*}(\mathbf{y}) d \mathbf{y} \\
& +\int_{\partial \Omega} G(\mathbf{y}-\mathbf{x})\left[\boldsymbol{\nu} \cdot \mathbf{q}^{*}(\mathbf{y})\right] d \mathbf{y} .
\end{aligned}
$$

In this equation, $\nu$ represents the outward unit vector normal to the remote boundary $\partial \Omega$ of $\Omega$.

Due to the fact that the Green's function $G(\mathbf{y}-\mathbf{x})$ is equal to zero for $\mathbf{y} \in \partial \boldsymbol{\Omega}$ and by using Eq. (21) of $\mathbf{q}^{*}$, it follows from Eq. (26) that

$$
T(\mathbf{x})=K_{i j}^{0} e_{j}^{0} \int_{\omega} G_{. i}(\mathbf{y}-\mathbf{x}) d \mathbf{y}+\int_{\Gamma} G(\mathbf{y}-\mathbf{x}) \operatorname{div}_{s} \mathbf{q}^{s}(\mathbf{y}) d \mathbf{y} .
$$

Correspondingly, the resulting intensity field components are given by

$$
\begin{aligned}
e_{m}(\mathbf{x}) & =-\frac{\partial T(\mathbf{x})}{\partial x_{m}} \\
& =K_{i j}^{0} e_{j}^{0} \int_{\omega} G_{, i m}(\mathbf{y}-\mathbf{x}) d \mathbf{y}+\int_{\Gamma} G_{, m}(\mathbf{y}-\mathbf{x}) \operatorname{div}_{s} \mathbf{q}^{s}(\mathbf{y}) d \mathbf{y}
\end{aligned}
$$

Now, let us show that this intensity field within a spherical or circular inclusion is, as in the case of perfect interfaces, independent of the position $\mathbf{x}$. To this aim, we first compute the right member of Eqs. (27) and (28) for a constant intensity field $\mathbf{e}(x)=e^{\omega}$ within the inclusion.

In the important special case where $\omega$ is a spherical or circular inclusion of radius $R$ and the intensity field $\mathrm{e}^{\omega}$ is uniform inside $\omega$, it is shown in the appendix that

$$
\operatorname{div}_{s} \mathbf{q}^{s}=-(d-1) \hat{k}_{s} \mathrm{e}^{\omega} \cdot \mathbf{n},
$$

where $\hat{k}_{s}=k_{s} / R$ and $d=3$ or 2 according as the $3 \mathrm{D}$ spherical or $2 \mathrm{D}$ circular inclusion is concerned.

Let us now compute the temperature field $T^{w}$ produced when a constant intensity field $e^{\omega}$ is present within the inclusion. Substituting Eq. (29) into Eq. (27) and using the divergence theorem, we obtain the temperature field $T^{\omega}$ relative to the constant intensity field $\mathbf{e}^{\omega}$

$$
\begin{aligned}
T^{\omega}(\mathbf{x})= & K_{i j}^{0} e_{j}^{0} \int_{\omega} G_{j}(\mathbf{y}-\mathbf{x}) d \mathbf{y}-(d-1) \hat{k}_{s} \\
& \times \int_{\omega}\left[G_{, i}(\mathbf{y}-\mathbf{x}) e_{i}^{\omega}+G(\mathbf{y}-\mathbf{x}) e_{i, j}^{\omega}\right] d \mathbf{y} .
\end{aligned}
$$

Taking into account the fact that the divergence of $\mathrm{e}^{\omega}$ is null inside $\omega$, the resulting intensity components are given by 


$$
\begin{aligned}
e_{m}^{\prime}(\mathbf{x}) & =-\frac{\partial T^{\omega}(\mathbf{x})}{\partial x_{m}} \\
& =K_{i j}^{0} e_{j}^{0} \int_{\omega} G_{i m}(\mathbf{y}-\mathbf{x}) d \mathbf{y}-(d-1) \hat{k}_{s} e_{i}^{\omega} \int_{\omega} G_{. i m}(\mathbf{y}-\mathbf{x}) d \mathbf{y} .
\end{aligned}
$$

If the intensity field is constant within the inclusion, the components $e_{m}^{\prime}$ must be identical to $e_{m}^{\omega}$ for any $\mathbf{x}$ inside $\omega$. Thus, for any $\mathbf{x}$ inside $\omega$, we have

$$
e_{m}^{\omega}=K_{i j}^{0} e_{j}^{0} \int_{\omega} G_{i m}(\mathbf{y}-\mathbf{x}) d \mathbf{y}-(d-1) \hat{k}_{s} e_{i}^{\omega} \int_{\omega} G_{, i m}(\mathbf{y}-\mathbf{x}) d \mathbf{y} .
$$

This last equation has an obvious solution given by

$$
\mathbf{e}^{\omega}=\overline{\mathrm{S}}^{\omega} \mathbf{e}^{0},
$$

where

$$
\overline{\mathbf{S}}^{\omega}=\left[\mathbf{I}^{(d)}+(d-1) \hat{k}_{s} S^{\omega} \mathbf{H}^{0}\right]^{-1} \mathbf{S}^{\omega} .
$$

Here, $\mathbf{S}^{\omega}$ stands for the classical Eshelby's conduction tensor inside $\omega$ without interface effect while $\overline{\mathbf{S}}^{\boldsymbol{\omega}}$ is defined as the Eshelby's conduction tensor inside $\omega$ with $\mathrm{HC}$ imperfect interface. Indeed, the classical Eshelby's conduction tensor fields both inside and outside $\omega$ with perfect interface are, in general, given by (see, e.g., Le-Quang et al. ${ }^{22}$ )

$$
\mathbf{S}(\mathbf{x})=\left[\int_{\omega} \boldsymbol{\nabla} \otimes \nabla G(\mathbf{y}-\mathbf{x}) d \mathbf{y}\right] \mathbf{K}^{0}
$$

From the previous set of equations, the general solution to the initial Eq. (28), both inside and outside $\omega$, is determined by combining Eqs. (29), (33), and (34) with Eq. (28) such that

$$
\mathbf{e}(\mathbf{x})=\overline{\mathbf{S}}(\mathbf{x}) \mathbf{e}^{0},
$$

where the tensor $\overline{\mathbf{S}}(\mathbf{x})$, called thereafter Eshelby's conduction tensor field with $\mathrm{HC}$ imperfect interface, is defined as

$$
\overline{\mathbf{S}}(\mathbf{x})=\mathbf{S}(\mathbf{x})-(d-1) \hat{k}_{s} \mathbf{S}(\mathbf{x}) \mathbf{H}^{0} \mathbf{S}^{\omega},
$$

where $\overline{\mathbf{S}}^{\omega}$ is given by Eq. (34).

As for the case of perfect interfaces, we remark from Eq. (37) that the Eshelby's conduction tensor fields with $\mathrm{HC}$ imperfect interface $\overline{\mathbf{S}}(\mathbf{x})$ given by Eq. (37) is uniform inside the inclusion $\omega$ and not uniform outside the inclusion $\omega$. Furthermore, $\overline{\mathbf{S}}(\mathbf{x})$ depends not only on the geometry of $\omega$ and on the material thermal anisotropy but also on the interface thermal properties $k_{s}$ and on the inclusion size $R$. It is interesting to note that when $k_{s}=0$ or when the size $R$ of heterogeneity is large, the surface effect becomes negligible and expression (37) of $\overline{\mathbf{S}}(\mathbf{x})$ reduces to formula (35) of the classical Eshelby's conduction tensor fields without interface effects. Moreover, we emphasize that the property that $\overline{\mathbf{S}}(\mathbf{x})$ is uniform inside the inclusion does not hold in the context of elasticity. More precisely, Shama and $\mathrm{Ganti}^{17}$ and Duan et al. ${ }^{18}$ have shown that the Eshelby's elastic tensor field is, in general, a quadratic function of the position coordinates and so is not uniform inside the inclusion.

Finally, we recall that the previous properties of the Eshelby's conduction tensor fields $\overline{\mathbf{S}}(\mathbf{x})$ with $\mathrm{HC}$ imperfect interface hold for any thermal anisotropy of the material forming $\Omega$. Thus, in order to obtain explicitly the expression of $\overline{\mathbf{S}}(\mathbf{x})$, the following particular cases are studied in detail.

\section{A. Isotropic media}

We are concerned with the important special case where the $2 \mathrm{D}$ or $3 \mathrm{D}$ infinite body $\Omega$ consists of an isotropic material with the themal-conductivity tensor given by $\mathbf{K}^{0}=k_{0} \mathbf{I}^{(d)}$, where $k_{0}$ is a positive scalar. Correspondingly, the Green's function $G(\mathbf{y}-\mathbf{x})$ takes the following form:

$$
G(\mathbf{y}-\mathbf{x})=-\frac{1}{4 \pi k_{0}|\mathbf{y}-\mathbf{x}|},
$$

when $\Omega$ is $3 \mathrm{D}$ and

$$
G(\mathbf{y}-\mathbf{x})=-\frac{1}{2 \pi k_{0}} \ln \frac{1}{|\mathbf{y}-\mathbf{x}|},
$$

when $\Omega$ is $2 \mathrm{D}$. In the foregoing expressions, $|\mathbf{y}-\mathbf{x}|$ denotes the Cartesian norm of $\mathbf{y}-\mathbf{x}$. By introducing the ordinary potential $\varphi(\mathbf{x})$ defined as

$$
\varphi(\mathbf{x})=k_{0} \int_{\omega} G(\mathbf{y}-\mathbf{x}) d \mathbf{y},
$$

which verifies

$$
\Delta \varphi(\mathbf{x})=\chi^{\omega}(\mathbf{x}),
$$

the classical Eshelby's elastic tensor fields $\mathbf{S}(\mathbf{x})$ is thus calculated by

$$
S_{i j}(\mathbf{x})=\frac{\partial^{2} \varphi(\mathbf{x})}{\partial x_{i} \partial x_{j}} .
$$

For a spherical or circular inclusion of radius $R$, the expression of $\varphi(\mathbf{x})$ can be explicitly determined by (see, e.g., Eshelby ${ }^{23}$ )

$$
\varphi(\mathbf{x})=\left\{\begin{array}{cc}
\frac{1}{6} r^{2}-\frac{1}{2} R^{2} & \text { for } r=|\mathbf{x}| \leq R, \\
-\frac{R^{3}}{3 r} & \text { for } r=|\mathbf{x}|>R,
\end{array}\right.
$$

when $\Omega$ is $3 \mathrm{D}$ and

$$
\varphi(\mathbf{x})=\left\{\begin{array}{cl}
\frac{1}{4} r^{2}+\frac{1}{2} R^{2}\left(\ln R-\frac{1}{2}\right) & \text { for } r=|\mathbf{x}| \leq R, \\
\frac{1}{2} R^{2} \ln r & \text { for } r=|\mathbf{x}|>R,
\end{array}\right.
$$

when $\Omega$ is $2 \mathrm{D}$.

Substituting Eqs. (43) and (44) into Eq. (42), the classical Eshelby's conduction tensor field reads 


$$
\mathbf{S}(\mathbf{x})=\frac{1}{d} \mathbf{I}^{(d)} \chi^{\omega}(\mathbf{x})+\left(1-\chi^{\omega}(\mathbf{x})\right) \xi^{d}\left(\frac{1}{d} \mathbf{I}^{(d)}-\overline{\mathbf{x}} \otimes \overline{\mathbf{x}}\right),
$$

where

$$
\xi=\frac{R}{r}=\frac{R}{|\mathbf{x}|}, \quad \overline{\mathbf{x}}=\frac{\mathbf{x}}{r} .
$$

By substituting Eq. (45) into Eq. (37), the expression of the Eshelby's conduction tensor field with $\mathrm{HC}$ imperfect interface for the isotropic medium is written as

$$
\overline{\mathbf{S}}(\mathbf{x})=\frac{1}{d(1+D)} \mathbf{I}^{(d)} \chi^{\omega}(\mathbf{x})+\left(1-\chi^{\omega}(\mathbf{x})\right) \frac{\xi^{d}}{1+D}\left(\frac{1}{d} \mathbf{I}^{(d)}-\overline{\mathbf{x}} \otimes \overline{\mathbf{x}}\right),
$$

where

$$
D=\frac{(d-1)}{d} \frac{\hat{k}_{s}}{k_{0}}
$$

\section{B. Anisotropic media}

We consider now the case where the medium $\Omega$ under consideration consists of a $2 \mathrm{D}$ or $3 \mathrm{D}$ anisotropic material. Without loss of generality, the thermal-conductivity tensor $\mathbf{K}^{0}$ can be written as

$$
\mathbf{K}^{0}=k_{0} \sum_{i=1}^{d} c_{i}^{-2} \mathbf{f}_{i} \otimes \mathbf{f}_{i},
$$

where $d$ is the dimension of $\Omega, k_{0}=\left(\operatorname{det} \mathbf{K}^{0}\right)^{1 / d}$ and $c_{i}$ are scalars such that $c_{1} c_{2}=1$ and $c_{1} \geq c_{2}$ or $c_{1} c_{2} c_{3}=1$ and $c_{1}$ $\geq c_{2} \geq c_{3}$ for the case $2 \mathrm{D}$ or $3 \mathrm{D}$, respectively. The Green's function $G(\mathbf{y}-\mathbf{x})$ being the solution to Eq. (25), or equivalently,

$$
\delta(\mathbf{y}-\mathbf{x})=k_{0} \sum_{i=1}^{d} c_{i}^{-2} G_{, i i}(\mathbf{y}-\mathbf{x})
$$

takes the following form:

$$
G(\mathbf{y}-\mathbf{x})=-\frac{1}{4 \pi k_{0} \sqrt{\sum_{i=1}^{3} c_{i}^{2}\left(y_{i}-x_{i}\right)^{2}}},
$$

when $\Omega$ is $3 \mathrm{D}$ and

$$
G(\mathbf{y}-\mathbf{x})=-\frac{1}{2 \pi k_{0}} \ln \frac{1}{\sqrt{\sum_{i=1}^{2} c_{i}^{2}\left(y_{i}-x_{i}\right)^{2}}},
$$

when $\Omega$ is $2 \mathrm{D}$. Setting $\tilde{x}_{i}=c_{i} x_{i}$ and $\tilde{y}_{i}=c_{i} y_{i}$ and denoting the partial derivative of $\mathbf{0}$ with respect to $\tilde{y}_{i}$ by $(\boldsymbol{Q})_{i}^{-}$, the components of the classical Eshelby's conduction tensor field are thus given by

$$
\begin{aligned}
S_{i j}(\mathbf{x}) & =K_{k j}^{0} \int_{\omega} G_{, j k}(\mathbf{y}-\mathbf{x}) d \mathbf{y} \\
& =\frac{c_{j}}{c_{i}} k_{0} \int_{\tilde{\omega}} \tilde{G}_{. i j}(\tilde{\mathbf{y}}-\tilde{\mathbf{x}}) d \tilde{\mathbf{y}} \\
& =\frac{c_{j}}{c_{i}} \tilde{S}_{i j}(\mathbf{x}) .
\end{aligned}
$$

Here, the summation convention rule does not apply for in$\operatorname{dex} i$ and $j$ and $\tilde{G}(\tilde{\mathbf{y}}-\tilde{\mathbf{x}})$ stands for the Green's function associated to a homogeneous isotropic medium with the thermal conductivity $k_{0}$ which is given by

$$
\tilde{G}(\mathbf{y}-x)=-\frac{1}{4 \pi k_{0}|\tilde{y}-\tilde{x}|}
$$

or

$$
\tilde{G}(\tilde{\mathbf{y}}-\tilde{\mathbf{x}})=-\frac{1}{2 \pi k_{0}} \ln \frac{1}{|\tilde{y}-\tilde{x}|}
$$

for the case $3 \mathrm{D}$ or $2 \mathrm{D}$, respectively; $\tilde{S}_{i j}(\tilde{\mathbf{x}})$ stands for the components of the classical Eshelby's conduction tensor field for an ellipsoidal inclusion with three principal axes $2 c_{1} R$, $2 c_{2} R$, and $2 c_{3} R$ in $3 \mathrm{D}$ or for an elliptical inclusion with two principal axes $2 c_{1} R$ and $2 c_{2} R$ in $2 \mathrm{D}$ embedded in the infinity homogeneous isotropic medium with the themal conductivity $k_{0}$.

First, using the well-known uniform classical Eshelby's conduction tensor inside the ellipsoidal or elliptical inclusion $\omega$ (see Eshelby ${ }^{23}$ ) and accounting for the relation (53), the explicit expression of the classical Eshelby's conduction tensor within $\omega$ for a $3 \mathrm{D}$ or $2 \mathrm{D}$ anisotropic medium reads

$$
\mathbf{S}^{\omega}=\widetilde{\mathbf{S}} \bar{\omega}=\sum_{i=1}^{d} J_{i} \mathbf{f}_{i} \otimes \mathbf{f}_{i},
$$

where (i) for the case where $\Omega$ is $3 \mathrm{D}$

$$
J_{1}=\frac{1}{\left(c_{1}^{2}-c_{2}^{2}\right)\left(c_{1}^{2}-c_{3}^{2}\right)^{1 / 2}}[F(\theta, m)-E(\theta, m)],
$$

$$
\begin{gathered}
J_{2}=\frac{1}{\left(c_{2}^{2}-c_{3}^{2}\right)\left(c_{1}^{2}-c_{3}^{2}\right)^{1 / 2}}\left\{\frac{c_{2}\left(c_{1}^{2}-c_{3}^{2}\right)^{1 / 2}}{c_{1} c_{3}}-E(\theta, m)\right\}, \\
J_{3}=1-J_{1}-J_{2}
\end{gathered}
$$

with $F(\theta, m)$ and $E(\theta, m)$ being elliptic integrals of the first and second kinds of amplitude and modulus

$$
\theta=\sin ^{-1}\left(1-c_{3}^{2} / c_{1}^{2}\right)^{1 / 2}, \quad m=\left(c_{1}^{2}-c_{2}^{2}\right)^{1 / 2} /\left(c_{1}^{2}-c_{3}^{2}\right)^{1 / 2},
$$

(ii) for the case where $\Omega$ is $2 \mathrm{D}$

$$
J_{1}=\frac{c_{2}}{c_{1}+c_{2}}, \quad J_{2}=\frac{c_{1}}{c_{1}+c_{2}} .
$$

Substituting Eq. (56) together with Eqs. (57)-(59) into Eq. (34) leads to the expression of the Eshelby's conduction ten- 
sor fields inside $\omega$ with HC imperfect interface for an anisotropic medium in $3 \mathrm{D}$ or $2 \mathrm{D}$,

$$
\overline{\mathbf{S}}^{\omega}=\sum_{i=1}^{d} \frac{J_{i}}{1+d D J_{i} c_{i}^{2}} \mathbf{f}_{i} \otimes \mathbf{f}_{i},
$$

where $D$ is given by Eq. (48).

It is easy to check that when the material within $\Omega$ is isotropic, or equivalently, $c_{i}=1$ and $J_{i}=1 / d$, the expression of $\overline{\mathbf{S}}^{\omega}$ given by Eq. (60) reduces to $\mathrm{Eq}$. (47) for any $\mathbf{x} \in \omega$.

Second, outside the ellipsoidal or elliptical inclusion $\tilde{\omega}$ the classical Eshelby's conduction tensor fields for a 3D or 2D anisotropic medium given by Eq. (53) with

$$
\tilde{S}_{i j}(\tilde{\mathbf{x}})=\frac{\partial^{2} \tilde{\varphi}(\tilde{\mathbf{x}})}{\partial \tilde{x}_{i} \partial \tilde{x}_{j}},
$$

where the ordinary potential $\tilde{\varphi}(\tilde{\mathbf{X}})$ is defined as

$$
\tilde{\varphi}(\tilde{\mathbf{x}})=k_{0} \int_{\bar{\omega}} \tilde{G}(\tilde{\mathbf{y}}-\tilde{\mathbf{x}}) d \tilde{\mathbf{y}} .
$$

More precisely, the expression of $\tilde{\varphi}(\tilde{\mathbf{X}})$ can be derived by:

(i) when $\Omega$ is $3 \mathrm{D}$ (see Kellogg, ${ }^{24}$ MacMillan, ${ }^{25}$ and Eshelby ${ }^{23}$ )

$$
\begin{aligned}
\tilde{\varphi}= & -\frac{R^{3}}{2 l^{3}}\left[l^{2}-\frac{\hat{x}_{1}^{2}}{m^{2}}+\frac{\hat{x}_{2}^{2}}{m^{2}}\right] F(\theta, m)-\frac{R^{3}}{2 l^{3}}\left[\frac{\hat{x}_{1}^{2}}{m^{2}}-\frac{\hat{x}_{2}^{2}}{m^{2} m^{\prime 2}}\right. \\
& \left.+\frac{\hat{x}_{3}^{2}}{m^{\prime 2}}\right] E(\theta, m)-\frac{R^{3}}{2 l^{2} m^{\prime 2}}\left[\frac{C}{A B} \hat{x}_{2}^{2}-\frac{B}{A C} \tilde{x}_{3}^{2}\right],
\end{aligned}
$$

where

$$
\begin{gathered}
A=\left(c_{1}^{2} R^{2}+\lambda\right)^{1 / 2}, \quad B=\left(c_{2}^{2} R^{2}+\lambda\right)^{1 / 2}, \\
C=\left(c_{3}^{2} R^{2}+\lambda\right)^{1 / 2}, \quad l=R\left(c_{1}^{2}-c_{3}^{2}\right)^{1 / 2}, \\
m^{2}=1-m^{\prime 2}=\frac{c_{1}^{2}-c_{2}^{2}}{c_{1}^{2}-c_{3}^{2}}, \quad \theta=\sin ^{-1}(l / A),
\end{gathered}
$$

and $\lambda$ is the positive root of

$$
\frac{\hat{x}_{1}^{2}}{A^{2}}+\frac{\hat{x}_{2}^{2}}{B^{2}}+\frac{\hat{x}_{3}^{2}}{C^{2}}=1,
$$

(ii) when $\Omega$ is $2 \mathrm{D}$ (see Ferrers, ${ }^{26}$ Dyson, ${ }^{27}$ and Beom ${ }^{28}$ )

$$
\bar{\varphi}=-\frac{1}{2} R^{2}\left\{J(\lambda)-\tilde{x}_{1}^{2} J_{1}(\lambda)-\tilde{x}_{2}^{2} J_{2}(\lambda)\right\},
$$

where

$$
\begin{gathered}
J(\lambda)=-\ln \left\{\frac{1}{2}\left(c_{1}^{2} R^{2}+\lambda\right)^{1 / 2}+\frac{1}{2}\left(c_{2}^{2} R^{2}+\lambda\right)^{1 / 2}\right\}+\frac{1}{2}, \\
J_{1}(\lambda)=\left(c_{1}^{2} R^{2}+\lambda\right)^{-1 / 2}\left[\left(c_{2}^{2} R^{2}+\lambda\right)^{1 / 2}+\left(c_{1}^{2} R^{2}+\lambda\right)^{1 / 2}\right]^{-1}, \\
J_{2}(\lambda)=\left(c_{2}^{2} R^{2}+\lambda\right)^{-1 / 2}\left[\left(c_{2}^{2} R^{2}+\lambda\right)^{1 / 2}+\left(c_{1}^{2} R^{2}+\lambda\right)^{1 / 2}\right]^{-1},
\end{gathered}
$$

and $\lambda$ is the positive root of

$$
\frac{\tilde{x}_{1}^{2}}{c_{1}^{2} R^{2}+\lambda}+\frac{\hat{x}_{2}^{2}}{c_{2}^{2} R^{2}+\lambda}=1 .
$$

Introducing $\mathbf{S}(\mathbf{x})$ given by Eq. (53) together with Eqs. (61)-(68) into Eq. (37), we obtain the expression of the Eshelby's conduction tensor fields outside $\omega$ with $\mathrm{HC}$ imperfect interface of a 3D or 2D anisotropic medium. Finally, it is interesting to note that this expression of $\overline{\mathbf{S}}(\mathbf{x})$ outside $\omega$ can be also used within $\omega$ by setting $\lambda=0$.

\section{SIZE-DEPENDENT LOCALIZATION TENSOR FIELDS}

We consider now the heterogeneity problem in which the inclusion $\omega$ embedded in the 2D or 3D infinity extended matrix domain $\Omega$ with the thermal conductivity and resistivity tensors, $\mathbf{K}^{0}$ and $\mathbf{H}^{0}$, is made of another material with the thermal-conductivity and resistivity tensors denoted by $\mathbf{K}^{(I)}$ and $\mathbf{H}^{(I)}$, respectively. As before, the interface $\Gamma$ between the matrix and inclusion is also assumed to be $\mathrm{HC}$. On the boundary $2 \Omega$ of $\Omega$, the following homogeneous intensity boundary condition is prescribed:

$$
T^{0}(\mathbf{x})=-\mathbf{e}^{0} \cdot \mathbf{x},
$$

where $\mathbf{x} \in \partial \Omega$ and $\mathbf{e}^{0}$ is a constant intensity field.

Similarly to the results obtained in Sec. III for the Eshelby's conduction tensor with $\mathrm{HC}$ imperfect interface, it can be shown that in this heterogeneity problem the intensity field is also uniform within a spherical or circular heterogeneity, i.e., $\mathbf{e}(\mathbf{x})=\mathbf{e}^{\omega}$ with $\mathbf{x} \in \omega$. In addition, the initial heterogeneity problem can be decomposed into three subproblems, denoted as A, B, and C (see Fig. 3). Each subproblem and its solution are then presented in detail as follows.

\section{A. Subproblem A}

This subproblem consists in computing the intensity solution field within the infinite heterogeneous matrix medium $\Omega$ with the thermal-conductivity and resistivity tensors, $\mathbf{K}^{0}$ and $\mathbf{H}^{0}$, subjected to the uniform intensity boundary condition [Eq. (69)] on its external surface $\partial \Omega$. Clearly, the corresponding intensity solution field $\mathbf{e}^{(A)}(\mathbf{x})$ is provided directly by

$$
\mathbf{e}^{(A)}(\mathbf{x})=\mathrm{e}^{0}
$$

for any $\mathbf{x} \in \Omega$.

\section{B. Subproblem B}

Subproblem B is concerned with the infinite heterogeneous matrix medium $\Omega$ having the thermal-conductivity and resistivity tensors, $\mathbf{K}^{0}$ and $\mathbf{H}^{\delta}$ in which the subdomain $\omega$ made of the same material as the matrix and surrounded by a HC imperfect interface $\Gamma$ is exposed to a uniform heat-free intensity field $\mathrm{e}^{*}=\left(\bar{S}^{\omega}\right)^{-1} \mathrm{e}^{\omega}$. Applying the Eshelby's conductivity tensor fields with $\mathrm{HC}$ imperfect interface obtained in Sec. III, the intensity solution field $\mathbf{e}^{(B)}(\mathbf{x})$ of subproblem B is given by

$$
\mathbf{e}^{(B)}(\mathbf{x})=\overline{\mathbf{S}}(\mathbf{x})\left(\overline{\mathbf{S}}^{\omega}\right)^{-1} \mathrm{e}^{\omega},
$$

where $\mathbf{x} \in \Omega$. 


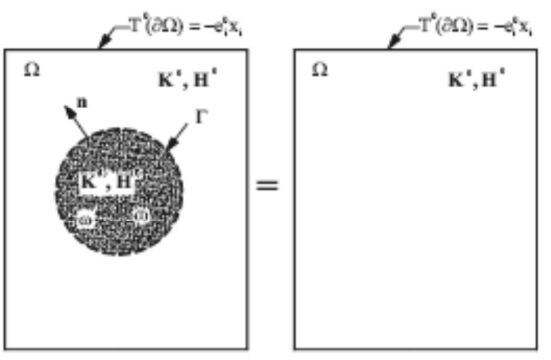

(a)

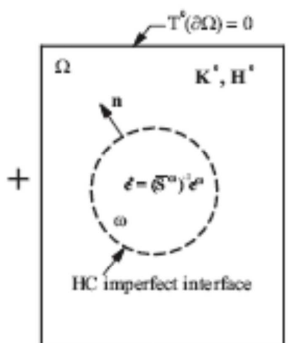

(b)

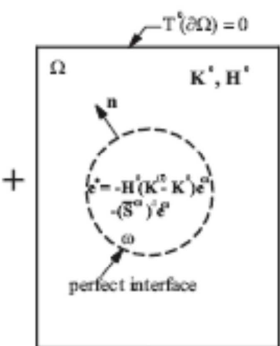

(c)
FG. 3. Decomposition of the initial heterogeneity problem into three subproblems (a)-(c) with $e^{\omega}$ being uniform intensity solution field within $\omega$ of the initial heterogeneity problem.

\section{Subproblem C}

In subproblem $\mathrm{C}$, we consider the subdomain $\omega$ embedded in the infinite heterogeneous matrix medium $\Omega$ with the thermal-conductivity and resistivity tensors, $\mathbf{K}^{0}$ and $\mathbf{H}^{0}$. Additionally, the subdomain $\omega$ is formed of the same material as the matrix and the interface between $\omega$ and the external medium is assumed to be perfect. Then, we let the subdomain $\omega$ undergo a uniform heat-free intensity field $\mathbf{e}^{*}=-\mathbf{H}^{0}\left(\mathbf{K}^{(I)}\right.$ $\left.-\mathbf{K}^{0}\right) \mathbf{e}^{\omega}-\left(\overline{\mathbf{S}}^{\omega}\right)^{-1} \mathbf{e}^{\omega}$. Due to the fact that the interface between $\omega$ and external medium is perfect and using the classical Eshelby's conductivity tensor field, the intensity solution field $\mathrm{e}^{(C)}(\mathrm{x})$ of subproblem $\mathrm{C}$ is then determined by

$$
\mathrm{e}^{(C)}(\mathbf{x})=-\mathbf{S}(\mathbf{x}) \mathbf{H}^{0}\left(\mathbf{K}^{(l)}-\mathbf{K}^{0}\right) \mathbf{e}^{\omega}-\mathbf{S}(\mathbf{x})\left(\overline{\mathbf{S}}^{\omega}\right)^{-1} \mathrm{e}^{\omega},
$$

for any $\mathbf{x} \in \Omega$.

Finally, by applying the superposition principle, the intensity solution field of the initial heterogeneity problem is given by

$$
\begin{aligned}
\mathrm{e}(\mathrm{x})= & \mathrm{e}^{(A)}(\mathrm{x})+\mathrm{e}^{(B)}(\mathrm{x})+\mathrm{e}^{(C)}(\mathrm{x}) \\
= & \mathrm{e}^{0}+\overline{\mathrm{S}}(\mathbf{x})\left(\overline{\mathbf{S}}^{\omega}\right)^{-1} \mathrm{e}^{\omega}-\mathrm{S}(\mathrm{x}) \mathbf{H}^{0}\left(\mathbf{K}^{(I)}-\mathbf{K}^{0}\right) \mathrm{e}^{\omega}-\mathrm{S}(\mathbf{x}) \\
& \times\left(\overline{\mathbf{S}}^{\omega}\right)^{-1} \mathrm{e}^{\omega},
\end{aligned}
$$

where $\mathbf{x} \in \Omega$.

Moreover, when $\mathbf{x} \in \omega$, it is immediate from the previous assumption that $\mathbf{e}(\mathbf{x})=\mathbf{e}^{\omega}$. The latter equation together with Eq. (73) for $\mathbf{x} \in \omega$ allows us to calculate $e^{\omega}$ as follows:

$$
\mathbf{e}^{\omega}=\overline{\mathbf{A}}^{\omega} \mathbf{e}^{0},
$$

where $\overline{\mathbf{A}}^{\omega}$, called thereafter the localization tensor inside $\omega$ with $\mathrm{HC}$ imperfect interface, is defined as

$$
\overline{\mathbf{A}}^{\omega}=\left[\mathbf{S}^{\omega}\left(\overline{\mathbf{S}}^{\omega}\right)^{-1}+\mathbf{S}^{\omega} \mathbf{H}^{0}\left(\mathbf{K}^{(l)}-\mathbf{K}^{0}\right)\right]^{-1}
$$

with $\mathbf{S}^{\omega}$ and $\overline{\mathbf{S}}^{\omega}$ given by Eqs. (56) and (60), respectively.

Indeed, when $\mathrm{e}^{\boldsymbol{\omega}}$ is obtained, the general intensity solution field $\mathbf{e}(\mathbf{x})$ in Eq. (73) for both inside and outside $\omega$, is calculated by combining Eqs. (74) and (75) with Eq. (73) such that

$$
\mathbf{e}(\mathbf{x})=\overline{\mathbf{A}}(\mathbf{x}) \mathbf{e}^{0},
$$

$\overline{\mathbf{A}}(\mathbf{x})$, named the localization tensor field with $\mathrm{HC}$ imperfect interface, is given by

$\overline{\mathbf{A}}(\mathbf{x})=\mathbf{I}^{(d)}-\left\{\mathbf{S}(\mathbf{x}) \mathbf{H}^{0}\left(\mathbf{K}^{(l)}-\mathbf{K}^{0}\right)+[\mathbf{S}(\mathbf{x})-\overline{\mathbf{S}}(\mathbf{x})]\left(\overline{\mathbf{S}}^{\omega}\right)^{-1}\right\} \overline{\mathbf{A}}^{\omega}$,

where $\overline{\mathbf{A}}^{\omega}, \mathbf{S}(\mathbf{x}), \overline{\mathbf{S}}(\mathbf{x})$, and $\overline{\mathbf{S}}^{\omega}$ are provided by Eqs. (75), (53), (37), and (60).

As the Eshelby's conduction tensor fields with $\mathrm{HC}$ imperfect interface $\mathbf{S}(\mathbf{x})$ given by Eq. (37), $\overline{\mathbf{A}}(\mathbf{x})$ depends not only on the geometry of $\omega$ and on the material thermal allotropies of the inclusion and matrix phases but also on the interface thermal properties $k_{s}$ and on the size of the inclusion $R$. We can check that when $k_{s}=0$ or when the size $R$ of heterogeneity is large, expression (77) of $\overline{\mathbf{A}}(\mathbf{x})$ reduces to well-known classical localization tensor field $\mathbf{A}(\mathbf{x})$.

An important particular case is when the matrix and inclusion phases are both isotropic with the thermalconductivity tensors given by $\mathbf{K}^{0}=k_{0} \mathbf{I}^{(d)}$ and $\mathbf{K}^{(l)}=k_{l} \mathbf{I}^{(d)}$ with $k_{0}$ and $k_{I}$ being two positive scalars. Correspondingly, the localization tensor fields $\overline{\mathbf{A}}(\mathbf{x})$ reduces to

$$
\begin{aligned}
\overline{\mathbf{A}}(\mathbf{x})= & \frac{d k_{0}}{k_{I}+(d-1)\left(k_{0}+\hat{k}_{s}\right)} \mathbf{I}^{(d)} \chi^{\omega}(\mathbf{x})+\left(1-\chi^{\omega}(\mathbf{x})\right) \\
& \times\left\{\mathbf{I}^{(d)}-\frac{d \xi^{d}\left[k_{I}-k_{0}+(d-1) \hat{k}_{s}\right]}{k_{I}+(d-1)\left(k_{0}+\hat{k}_{s}\right)}\left(\frac{1}{d} \mathbf{I}^{(d)}-\overline{\mathbf{x}} \otimes \overline{\mathbf{x}}\right)\right\} .
\end{aligned}
$$

\section{EFFECTIVE THERMAL BEHAVIOR}

In this section, the closed-form expressions for the effective conductivity moduli of composites with highly conducting imperfect interfaces are deduced by using some results of the previous two sections and by applying the dilute, MoriTanaka, self-consistent, and generalized self-consistent schemes. For more details about these schemes in the case of perfect interfaces, the reader can refer to the two excellent review papers of Hashin. ${ }^{29,30}$

\section{A. Dilute distribution model}

We consider now the composite described in Sec. II. Assuming that the interaction between the inhomogeneities is neglected, a dilute distribution of inhomogeneities is then 
adopted to estimate the effective thermal-conductivity tensor $\mathbf{K}^{e f f}$. For this purpose, we first let $\Omega$ be subjected to the homogeneous intensity boundary conditions given by Eq (69).

Under the boundary condition [Eq. (69)], owing to the fact that the temperature field is continuous across the interface between the matrix and inclusions phases, the macroscopic intensity field defined by Eq. (13) is obtained immediately by

$$
E=\langle e\rangle=e^{0} .
$$

Within the framework of the dilute distribution model, the interaction between the inhomogeneities is omitted and accounting for the results obtained for the localization tensor fields in Sec. IV, it can be shown that the intensity and heat flux fields inside the inhomogeneity $\omega$ are uniform and given by

$$
\begin{gathered}
\overline{\mathbf{e}}^{(1)}=\left[\mathbf{S}^{\omega}\left(\overline{\mathbf{S}}^{\omega}\right)^{-1}+\mathbf{S}^{\omega} \mathbf{H}^{(2)}\left(\mathbf{K}^{(1)}-\mathbf{K}^{(2)}\right)\right]^{-1} \mathbf{e}^{0}, \\
\overline{\mathbf{q}}^{(1)}=\mathbf{K}^{(1)}\left[\mathbf{S}^{\omega}\left(\overline{\mathbf{S}}^{\omega}\right)^{-1}+\mathbf{S}^{\omega} \mathbf{H}^{(2)}\left(\mathbf{K}^{(1)}-\mathbf{K}^{(2)}\right)\right]^{-1} \mathbf{e}^{0} .
\end{gathered}
$$

Here, $\mathbf{K}^{(i)}$ and $\mathbf{H}^{(i)}$ are thermal-conductivity and resistivity second-order tensors of phase $i$, respectively; the Eshelby's conduction tensors without and with interface effects, $\mathbf{S}^{\omega}$ and $\overline{\mathbf{S}}^{\omega}$, inside $\omega$ are, respectively, determined by Eqs. (56) and (60) with $\mathbf{K}^{0}=\mathbf{K}^{(2)}$ and $\mathbf{H}^{0}=\mathbf{H}^{(2)}$.

From Eq. (79), the volume or surface averages of the intensity and heat flux fields in the matrix phase are given by

$$
\begin{aligned}
\overline{\mathbf{e}}^{(2)} & =c_{2}^{-1}\left(\mathbf{e}^{0}-c_{1} \overline{\mathbf{e}}^{(1)}\right) \\
& =c_{2}^{-1}\left\{\mathbf{I}^{(d)}-c_{1}\left[\mathbf{S}^{\omega}\left(\overline{\mathbf{S}}^{\omega}\right)^{-1}+\mathbf{S}^{\omega} \mathbf{H}^{(2)}\left(\mathbf{K}^{(1)}-\mathbf{K}^{(2)}\right)\right]^{-1}\right\} \mathbf{e}^{0},
\end{aligned}
$$

$$
\begin{aligned}
\overline{\mathbf{q}}^{(2)} & =\mathbf{K}^{(2)} \overline{\mathbf{e}}^{(2)} \\
& =c_{2}^{-1} \mathbf{K}^{(2)}\left\{\mathbf{I}^{(d)}-c_{1}\left[\mathbf{S}^{\omega}\left(\overline{\mathbf{S}}^{\omega}\right)^{-1}+\mathbf{S}^{\omega} \mathbf{H}^{(2)}\left(\mathbf{K}^{(1)}-\mathbf{K}^{(2)}\right)\right]^{-1}\right\} \mathbf{e}^{0},
\end{aligned}
$$

where $c_{1}$ and $c_{2}=1-c_{1}$ are the volume or surface fractions of the inclusion and matrix phases, respectively. The macroscopic heat flux field obtained from Eq. (14) is calculated by

$$
\mathbf{Q}=c_{1} \overline{\mathbf{q}}^{(1)}+c_{2} \overline{\mathbf{q}}^{(2)}+c_{1}(d-1) \hat{k}_{s} \overline{\mathbf{e}}^{(1)} .
$$

Unlike the classical case where the matrix-inclusion interface is perfect, the macroscopic heat flux given by Eq. (84) is not simply the volume or surface average of the counterparts over the domain $\Omega$. More precisely, a new term due to the discontinuity of the normal component of the heat flux field across the $\mathrm{HC}$ interface $\Gamma$ is added in Eq. (84). By introducing Eqs. (80)-(83) into Eq. (84) and by identifying the resulting expression with Eq. (12), we obtain the expression of the effective thermal-conductivity tensor

$$
\begin{aligned}
\mathbf{K}^{D D}= & \mathbf{K}^{(2)}+c_{1}\left[\mathbf{K}^{(1)}-\mathbf{K}^{(2)}+(d-1) \hat{k}_{s} \mathbf{I}^{(d)}\right] \\
& \times\left[\mathbf{S}^{\omega}\left(\overline{\mathbf{S}}^{\omega}\right)^{-1}+\mathbf{S}^{\omega} \mathbf{H}^{(2)}\left(\mathbf{K}^{(1)}-\mathbf{K}^{(2)}\right)\right]^{-1} .
\end{aligned}
$$

It is interesting to note that when $k_{s}=0$, expression (85) of $\mathbf{K}^{\text {eff }}$ reduces to the well-known formula without interface effects. In the particular case where the matrix and inclusion phases are isotropic and have the themal conductivities $k_{2}$ and $k_{1}$, respectively, and by assuming that the effective behavior is also isotropic, Eq. (85) leads to

$$
k^{D D}=k_{2}+\frac{c_{1} d k_{2}\left[k_{1}-k_{2}+(d-1) \hat{k}_{s}\right]}{k_{1}+(d-1)\left(k_{2}+\hat{k}_{s}\right)},
$$

where $k^{D D}$ is the effective thermal conductivity of the composite for the dilute distribution model.

\section{B. Mori-Tanaka model}

To account for the interaction between the heterogeneities, the Mori-Tanaka model is now applied. In this model, under the boundary conditions, Eq. (69), the intensity field in the matrix and far from the inclusions is assumed to be uniform and equal to $\mathbf{E}^{0}$. Consequently, the intensity and heat flux fields inside the inhomogeneities $\omega$ are obtained by using Eqs. (80) and (81) with $\mathbf{e}^{0}$ being replaced by $\mathbf{E}^{0}$. Thus, this yields

$$
\begin{gathered}
\overline{\mathbf{e}}^{(1)}=\left[\mathbf{S}^{\omega}\left(\overline{\mathbf{S}}^{\omega}\right)^{-1}+\mathbf{S}^{\omega} \mathbf{H}^{(2)}\left(\mathbf{K}^{(1)}-\mathbf{K}^{(2)}\right)\right]^{-1} \mathbf{E}^{0}, \\
\overline{\mathbf{q}}^{1}=\mathbf{K}^{(1)}\left[\mathbf{S}^{\omega}\left(\overline{\mathbf{S}}^{\omega}\right)^{-1}+\mathbf{S}^{\omega} \mathbf{H}^{(2)}\left(\mathbf{K}^{(1)}-\mathbf{K}^{(2)}\right)\right]^{-1} \mathbf{E}^{0},
\end{gathered}
$$

where the Eshelby's conduction tensors without and with interface effects, $\mathbf{S}^{\omega}$ and $\overline{\mathbf{S}}^{\omega}$, inside $\omega$ are, respectively, provided by Eqs. (56) and (60) with $\mathbf{K}^{0}=\mathbf{K}^{(2)}$ and $\mathbf{H}^{0}=\mathbf{H}^{(2)}$. Concerning the matrix phase, the volume or surface average intensity and heat flux fields are given by

$$
\overrightarrow{\mathbf{e}}^{(2)}=\mathbf{E}^{0}, \quad \overline{\mathbf{q}}^{(2)}=\mathbf{K}^{(2)} \mathbf{E}^{0} .
$$

Due to the fact that $\mathbf{E}=\langle\mathbf{e}\rangle=\mathbf{e}^{0}$, we can express $\mathbf{E}^{0}$ in the terms of the macroscopic intensity field as follows:

$$
\mathbf{E}^{0}=\mathbf{L} \mathbf{e}^{0}
$$

with

$$
\mathbf{L}=\left\{c_{1}\left[\mathbf{S}^{\omega}\left(\overline{\mathbf{S}}^{\omega}\right)^{-1}+\mathbf{S}^{\omega} \mathbf{H}^{(2)}\left(\mathbf{K}^{(1)}-\mathbf{K}^{(2)}\right)\right]^{-1}+c_{2} \mathbf{I}^{(d)}\right\}^{-1} .
$$

By substituting Eqs. (87)-(91) into Eq. (84) and by identifying the resulting equation with Eq. (12), the effective thermal-conductivity tensor is given by

$$
\mathbf{K}^{M T}=\mathbf{K}^{(1)}+(d-1) \hat{k}_{s} \mathbf{I}^{(d)}-c_{2}\left[\mathbf{K}^{(1)}-\mathbf{K}^{(2)}+(d-1) \hat{k}_{s} \mathbf{I}^{(d)}\right] \mathbf{L} .
$$

In the special case where the matrix and inclusion phases are isotropic and have the thermal conductivities $k_{2}$ and $k_{1}$, respectively, and by assuming that the effective behavior is also isotropic, we obtain from Eq. (92) the effective thermal conductivity of the composite for the Mori-Tanaka model

$$
k^{M T}=k_{2}+\frac{c_{1} d k_{2}\left[k_{1}-k_{2}+(d-1) \hat{k}_{s}\right]}{d k_{2}+c_{2}\left[k_{1}-k_{2}+(d-1) \hat{k}_{s}\right]} .
$$

It is easy to verify that when the volume or surface fraction of the inclusion is small enough $\left(c_{1} \ll 1\right)$, expression (93) of 
the effective thermal conductivity of the composite for the Mori-Tanaka model reduces to the one obtained by Eq. (86) for the dilute distribution model.

\section{Self-consistent mode}

As for all estimate schemes for the effective thermal behavior of the composite, it is necessary to determine the volume or surface average of the intensity and heat flux fields inside the inhomogeneities. In the self-consistent model, the intensity and heat flux fields inside each inhomogeneity is estimated by embedding this inhomogeneity in a fictitious infinite medium which has unknown effective properties. By using the expression for the localization problem for themal conduction described in Sec. IV, we can calculate the intensity and heat flux fields inside the inhomogeneity $\omega$ as follows:

$$
\begin{gathered}
\overline{\mathrm{e}}^{(1)}=\left[\mathrm{S}^{\omega}\left(\overline{\mathbf{S}}^{\omega}\right)^{-1}+\mathbf{S}^{\omega} \mathbf{H}^{S C}\left(\mathbf{K}^{(1)}-\mathbf{K}^{S C}\right)\right]^{-1} \mathrm{e}^{0}, \\
\overline{\mathbf{q}}^{(1)}=\mathbf{K}^{(1)}\left[\mathbf{S}^{\omega}\left(\overline{\mathbf{S}}^{\omega}\right)^{-1}+\mathbf{S}^{\omega} \mathbf{H}^{S C}\left(\mathbf{K}^{(1)}-\mathbf{K}^{S C}\right)\right]^{-1} \mathrm{e}^{0} .
\end{gathered}
$$

In these expressions, $\mathbf{S}^{\alpha}$ and $\overline{\mathbf{S}}^{\omega}$ are given by Eqs. (56) and $(60)$ in which $\mathbf{K}^{0}$ and $\mathbf{H}^{0}$ are replaced by the unknown effective thermal-conductivity and resistivity tensors, $\mathbf{K}^{S C}$ and $\mathbf{H}^{S C}$, respectively.

As in the dilute distribution model, owing to the fact that $E=\langle e\rangle=e^{0}$, the volume or surface averages of the intensity and heat flux fields in the matrix phase are determined by

$$
\begin{aligned}
\overline{\mathbf{e}}^{(2)} & =c_{2}^{-1}\left(\mathbf{e}^{0}-c_{1} \overline{\mathbf{e}}^{(2)}\right) \\
& =c_{2}^{-1}\left\{\mathbf{I}^{(d)}-c_{1}\left[\mathbf{S}^{\omega}\left(\overline{\mathbf{S}}^{\omega}\right)^{-1}+\mathbf{S}^{\omega} \mathbf{H}^{S C}\left(\mathbf{K}^{(1)}-\mathbf{K}^{S C}\right)\right]^{-1}\right\} \mathbf{e}^{0},
\end{aligned}
$$

$$
\begin{aligned}
\overline{\mathbf{q}}^{(2)} & =\mathbf{K}^{(2)} \overline{\mathbf{e}}^{(2)} \\
& =c_{2}^{-1} \mathbf{K}^{(2)}\left\{\mathbf{I}^{(d)}-c_{1}\left[\mathbf{S}^{\omega}\left(\overline{\mathbf{S}}^{\omega}\right)^{-1}+\mathbf{S}^{\omega} \mathbf{H}^{s c}\left(\mathbf{K}^{(1)}-\mathbf{K}^{S C}\right)\right]^{-1}\right\} \mathbf{e}^{0} .
\end{aligned}
$$

Introducing Eqs. (94)-(97) into Eq. (84) and accounting for the macroscopic behavior Eq. (12), we obtain an equation for the effective thermal-conductivity tensor

$$
\begin{aligned}
\mathbf{K}^{S C}= & \mathbf{K}^{(2)}+c_{1}\left[\mathbf{K}^{(1)}-\mathbf{K}^{(2)}+(d-1) \hat{k}_{s} \mathbf{I}^{(d)}\right]\left[\mathbf{S}^{\omega}\left(\overline{\mathbf{S}}^{\omega}\right)^{-1}\right. \\
& \left.+\mathbf{S}^{\omega} \mathbf{H}^{S C}\left(\mathbf{K}^{(1)}-\mathbf{K}^{S C}\right)\right]^{-1} .
\end{aligned}
$$

This second-order polynomial matrix equation provides the effective conductivity tensor $\mathbf{K}^{S C}$. If the matrix and inclusion phases are isotropic and characterized by the thermal conductivities $k_{2}$ and $k_{1}$ and if the effective behavior is assumed to be also isotropic, Eq. (98) is reduced to a quadratic equation

$$
k^{S C}=k_{2}+\frac{c_{1} d k^{S C}\left[k_{1}-k_{2}+(d-1) \hat{k}_{s}\right]}{k_{1}+(d-1)\left(k^{S C}+\hat{k}_{s}\right)}
$$

and the effective thermal conductivity $k^{S C}$ is calculated as the real positive root of Eq. (99).

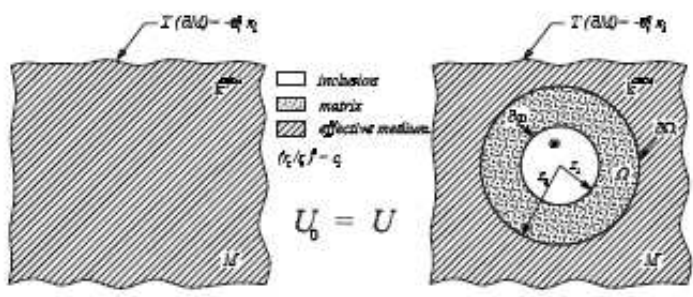

FIG. 4. Generalized self-consistent model applied to thermalconduction problem.

\section{Generalized self-consistent model}

The model proposed in this section does not use the Eshelby's tensor fields and localization tensor fields but is presented here in order to check the validity of the results obtained above. It can be considered as an extension to the thermal-conduction problem with interface effects of the classical generalized self-consistent model (GSCM) for elasticity which was initiated by Van der Poel, ${ }^{31}$ improved and completed by Smith ${ }^{32,33}$ and Christensen and Lo. ${ }^{34}$

According to this model, we first consider an infinite three-dimensional medium $M$ consisting of the effective homogeneous and isotropic medium whose themal behavior is characterized by Eq. (12) in which the effective thermalconductivity tensor is denoted by $\mathbf{K}^{e f f}=k^{\mathrm{GSCM}} \mathbf{I}^{(3)}$. As before, let $M$ now be subjected to the uniform boundary conditions, Eq. (69), in which the constant intensity vector $\mathrm{e}^{0}$ is chosen such as $\mathbf{e}^{0}=\left(0,0, e^{0}\right)^{T}$ with $e^{0}$ being a constant intensity field (Fig. 4). In the system of spherical coordinates $(r, \theta, \varphi)$ corresponding to the spherical orthogonal basis $\left(\mathbf{f}_{r}, \mathbf{f}_{\theta}, \mathbf{f}_{\varphi}\right)$, this uniform boundary condition takes the equivalent form

$$
T^{0}(\mathbf{x})=-e^{\theta} r \cos \theta, \quad \mathbf{x} \in \partial M .
$$

This boundary condition gives rise to the following temperature, intensity, and heat flux fields in $M$ :

$$
\begin{gathered}
T^{0}(\mathbf{x})=-e^{0} r \cos \theta, \quad \mathrm{e}^{0}(\mathbf{x})=e^{0}\left(\cos \theta \mathbf{f}_{r}-\sin \theta \mathbf{f}_{\theta}\right), \\
\mathbf{q}^{0}(\mathbf{x})=k^{\mathrm{GSCM}} e^{0}\left(\cos \theta \mathbf{f}_{r}-\sin \theta \mathbf{f}_{\theta}\right) .
\end{gathered}
$$

Let us introduce the following virtual work $U_{0}\left(\mathrm{e}^{0}\right)$ of $M$ (see, e.g., Hashin ${ }^{35}$ ):

$$
U_{0}\left(\mathbf{e}^{0}\right)=\int_{M} \mathbf{q}^{0}(\mathbf{x}) \cdot \mathbf{e}^{0}(\mathbf{x}) d \mathbf{x}=\operatorname{vol}(M) k^{\mathrm{GSCM}}\left(e^{0}\right)^{2} .
$$

Next, we cut a sphere out of the foregoing infinite effective medium and substitute back a composite sphere $\Omega$ while imposing the same boundary condition on $\partial M$ as before. The interface between the composite sphere and the outside medium is assumed to be perfect. The core of this composite sphere is made of the inclusion phase, referred to as phase 1 and surrounded by a concentric shell consisting of the matrix phase, denoted by phase 2 . The core and outer coating consist of two isotropic materials whose thermal conductivities are $k_{1}$ and $k_{2}$. The radii of the core and coating, symbolized 
by $r_{1}$ and $r_{2}$ are chosen so as to be compatible with the prescribed phase volume fraction

$$
c_{1}=1-c_{2}=\frac{r_{1}^{3}}{r_{2}^{3}} \text {. }
$$

The 3D spherical interface $\Gamma$ between the matrix and inclusion is modeled by the $\mathrm{HC}$ surface model as described in Sec. II.

Under the boundary condition, Eq. (100), the expressions of the temperature field, nonzero intensity, and heat flux field components are given by (see, e.g., Hervé ${ }^{36}$ )

$$
\begin{gathered}
T^{(i)}=-\left(a_{i} r+\frac{b_{i}}{r^{2}}\right) \cos \theta, \\
e_{r}^{(i)}=\left(a_{i}-\frac{2 b_{i}}{r^{3}}\right) \cos \theta, \quad e_{\theta}^{(i)}=-\left(a_{i}+\frac{b_{i}}{r^{3}}\right) \sin \theta, \\
q_{r}^{(i)}=k_{i}\left(a_{i}-\frac{2 b_{i}}{r^{3}}\right) \cos \theta, \quad q_{\theta}^{(i)}=-k_{i}\left(a_{i}+\frac{b_{i}}{r^{3}}\right) \sin \theta
\end{gathered}
$$

with $i=1$ referring to the core, $i=2$ to the outer coating and $i=e$ to the external effective medium. In these expressions, $a_{i}$ and $b_{i}$ are constants to be determined from the boundary and interface conditions together with a condition avoiding the displacement singularity in the core of the composite sphere. More precisely, the requirement of the temperature in the core at $r=0$ to be finite implies that $b_{1}=0$. The value of $a_{e}$, determined by using the boundary condition, Eq. (100), with $r \rightarrow \infty$, is given by $a_{c}=e^{0}$

At the interface between the core and the coating, the temperature and tangential part of the intensity fields are continuous so that

$$
\begin{gathered}
a_{1}=a_{2}+\frac{b_{2}}{r_{1}^{3}}, \\
e_{\theta}^{s}=e_{\theta}^{(1)}=e_{\theta}^{(2)}=-\left(a_{2}+\frac{b_{2}}{r_{1}^{3}}\right) \sin \theta, \\
e_{\varphi}^{s}=e_{\varphi}^{(1)}=e_{\varphi}^{(2)}=0 .
\end{gathered}
$$

The surface condition [Eq. (6)] at the interface between the core and the coating can be specified by accounting for Eqs. (5), (7), (108), and (104) and reduced to the following one:

$$
k_{2}\left(a_{2}-\frac{2 b_{2}}{r_{1}^{3}}\right)-k_{1} a_{1}=2 \hat{k}_{s}\left(a_{2}+\frac{b_{2}}{r_{1}^{3}}\right),
$$

with $\hat{k}_{s}=k_{s} / r_{1}$.

At the same time, the interface at $r=r_{2}$ between the coating matrix and outside effective medium is perfectly bonded. Thus, the continuity conditions of the temperature field $T$ and normal component heat flux field $q_{r}$ across the interface at $r=r_{2}$ are expressed as

$$
e^{0}+\frac{b_{c}}{r_{2}^{3}}=a_{2}+\frac{b_{2}}{r_{2}^{3}},
$$

$$
k^{\mathrm{GSCM}}\left(e^{0}-\frac{2 b_{e}}{r_{2}^{3}}\right)=k_{2}\left(a_{2}-\frac{2 b_{2}}{r_{2}^{3}}\right) .
$$

As in the GSCM of Christensen and Lo (1979) in the context of elasticity, the effective thermal conductivity is required to be such that the virtual work $U\left(\mathrm{e}^{0}\right)$ after introducing the sphere composite is equal to the initial one $U_{0}\left(\mathrm{e}^{0}\right)$ which is given by $\mathrm{Eq}$. (102). On the other hand, we can show that the actual virtual work $U\left(\mathrm{e}^{0}\right)$ can be expressed in the terms of $U_{0}\left(\mathrm{e}^{0}\right)$ as follows:

$$
U=U_{0}+\int_{\Gamma}\left(\mathbf{q}^{0} T^{(e)}-\mathbf{q}^{(e)} T^{0}\right) \cdot \mathbf{n} d \mathbf{x} .
$$

Thus, the self-consistency condition $U_{0}=U$ is reduced to

$$
\int_{\Gamma}\left(\mathbf{q}^{0} T^{(e)}-\mathbf{q}^{(e)} T^{0}\right) \cdot \mathbf{n} d \mathbf{x}=0 .
$$

Substituting Eqs. (100), (101), (104), and (106) with $i=e$ into Eq. (113), we obtain the simple equation

$$
b_{e}=0 \text {. }
$$

Finally, substituting $b_{e}=0$ into Eqs. (107) and (109)-(111), we obtain a system of four homogeneous linear equations for the four unknowns $a_{1}, a_{2}, b_{2}$, and $e^{0}$. A nontrivial solution to this system exists if and only if the determinant of the relevant $4 \times 4$ matrix is equal to zero. This necessary and sufficient condition yields the expression for the effective thermal conductivity as follows:

$$
k^{\mathrm{GSCM}}=k_{2}+\frac{3 k_{2} c_{1}\left(k_{1}-k_{2}+2 \hat{k}_{s}\right)}{3 k_{2}+c_{2}\left(k_{1}-k_{2}+2 \hat{k}_{s}\right)} .
$$

First, we remark that the effective thermal conductivity obtained by Eq. (115) depends not only on the phase thermal properties and volume fractions but also on the interface thermal conductivity and on the size of the inhomogeneities through $\hat{k}_{s^{*}}$ When setting $\hat{k}_{s}=0$, expression (115) of $k^{\mathrm{GSCM}}$ reduces to the one obtained by Hashin and Shtrikman ${ }^{37}$ or Hervé ${ }^{36}$ without interface effects.

Next, it is interesting to note that formula (115) for the effective thermal conductivity can be obtained by replacing the elastic moduli $\kappa_{1}, \kappa_{2}, \mu_{1}, \mu_{2}$, and $\kappa_{s}$ by $k_{1}, k_{2}, 3 k_{1} / 2$, $3 k_{2} / 2$, and $3 k_{s} / 2$, respectively, into Eq. (106) of Le Quang and $\mathrm{He}^{38}$ by setting $\alpha_{1}=\alpha_{2}=1$ for elasticity. This correspondence between the elasticity and thermal conduction was mentioned and exploited by Gilormini ${ }^{39}$ and $\mathrm{He}$ and Le-Quang. ${ }^{40}$

In the $2 \mathrm{D}$ case, the corresponding effective thermal conductivity is given by

$$
k^{\mathrm{GSCM}}=k_{2}+\frac{2 k_{2} c_{1}\left(k_{1}-k_{2}+\hat{k}_{s}\right)}{2 k_{2}+c_{2}\left(k_{1}-k_{2}+\hat{k}_{s}\right)} .
$$

The details of the derivation are omitted here.

Finally, it is convenient to express Eqs. (115) and (116) for $k^{\mathrm{GSCM}}$ by the following compact one: 


$$
k^{\mathrm{GSCM}}=k_{2}+\frac{d k_{2} c_{1}\left[k_{1}-k_{2}+(d-1) \hat{k}_{s}\right]}{d k_{2}+c_{2}\left[k_{1}-k_{2}+(d-1) \hat{k}_{s}\right]},
$$

where $d(=3$ or 2$)$ is the dimension of the problem. Compared with Eq. (93) for the case where the matrix and inclusion phases are isotropic, we note that in this case $k^{\mathrm{GSCM}}$ $=k^{M T}$.

\section{NUMERICAL EXAMPLES}

To numerically illustrate the features of the results obtained above, we now consider the cases where the circular inhomogeneities having the same radius $R$ are introduced into a $2 \mathrm{D}$ host matrix phase. The matrix and inclusion phases are assumed to be both isotropic. Additionally, the inclusion phase is supposed to be less conducting than the matrix one. The thermal conductivities of the matrix and of the inhomogeneities are such as

$$
k_{1}=0.1 \mathrm{~W} \mathrm{~m}^{-1} \mathrm{~K}^{-1}, \quad k_{2}=1 \mathrm{~W} \mathrm{~m}^{-1} \mathrm{~K}^{-1} .
$$

Concerning the thermal parameters of the interface, we start with the interphase model in which the intermediate medium between the inhomogeneities and matrix phases are assumed to be very thin and very highly conducting. The corresponding conductivity and thickness are chosen as:

case 1: $R=4 \mu \mathrm{m}, \quad k_{\text {inter }}=20 \mathrm{~W} \mathrm{~m}^{-1} \mathrm{~K}^{-1}, h=50 \mathrm{~nm}$,

case 2: $R=2 \mu \mathrm{m}, \quad k_{\text {inter }}=50 \mathrm{~W} \mathrm{~m}^{-1} \mathrm{~K}^{-1}, \quad h=20 \mathrm{~nm}$.

Then, from the asymptotic approach (see, e.g., Miloh and Benveniste, ${ }^{12}$ Hashin $^{9}$ and Benveniste ${ }^{14}$ ), it is known that the thin and highly conducting layer can be appropriately modeled as a HC imperfect interface. In this case, the surface thermal conductivity is thus given by

$$
k_{s}=h k_{\text {inser }}=10^{-6} \mathrm{~W} \mathrm{~m}^{-1} \mathrm{~K}^{-1} .
$$

In the first example, by applying an uniform intensity vector $\mathrm{e}^{0}=(-1,0)^{T}$ at the boundary of the heterogeneous medium consisting of a circular inclusion with radius $R$ $=4 \mu \mathrm{m}$ embedded in a square matrix $20 \mu \mathrm{m} \times 20 \mu \mathrm{m}$ and by assuming the interface between the matrix and inclusion to be $\mathrm{HC}$ imperfect with the surface thermal conductivity provided by Eq. (119), we calculate first the heat flux field in the inclusion and matrix phases. Then, these values obtained for the heat flux field are compared with the ones given by using the FEM with the three-phase model (matrix/ interphase/inclusion phases) described in Fig. 5. The conductivity and the thickness of the interphase take the values provided in case 1. It is seen from Fig. 6 that the heat flux field is uniform within the inclusion and it shows also a good agreement between the present analytical solution and the numerical solution by FEM inside the inclusion and within its neighborhood. However, we observe also from Fig. 6 that there is a difference for the heat flux field in the matrix phase between the present analytical solution and numerical solution by FEM. This difference is explained by the fact that the closed-form solution obtained in Sec. IV is applicable for an infinite matrix medium while the numerical solution by FEM is calculated for a square finite matrix. Moreover, this explanation can be clarified by studying the second example
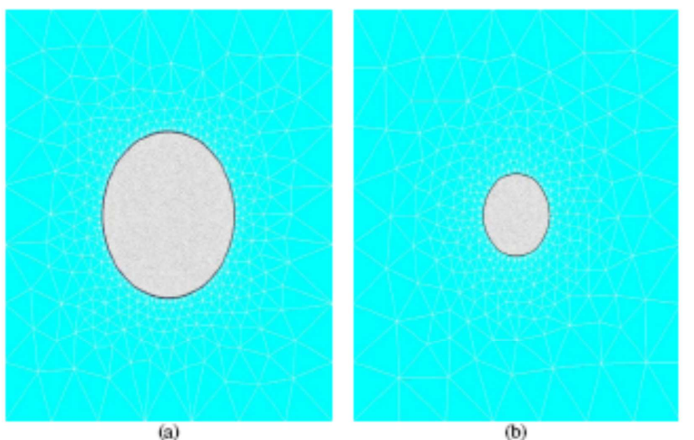

FIG. 5. (Color online) Solving the heterogeneity problem by FEM: (a) case 1 with inclusion radius $R=4 \mu \mathrm{m}$, thermal conductivity of the interphase $k_{\text {inter }}=20 \mathrm{~W} \mathrm{~m}^{-1} \mathrm{~K}^{-1}$ and thickness of the interphase $h=50 \mathrm{~nm}$; (b) case 2 with inclusion radius $R=2 \mu \mathrm{m}$, thermal conductivity of the interphase $k_{\text {inter }}=50 \mathrm{~W} \mathrm{~m}^{-1} \mathrm{~K}^{-1}$ and thickness of the interphase $h=20 \mathrm{~nm}$.

where the radius inclusion is reduced from 4 to $2 \mu \mathrm{m}$. In this example, the conductivity and the thickness of the interphase take the values given in case 2. As for the first example, we observe from Fig. 7 that the heat flux field is uniform in the inclusion. It can be seen from Fig. 7 that when the inclusion size is small, for example, $R=2 \mu \mathrm{m}$, the proposed analytical solution presents a good agreement with the numerical solution by FEM both inside and outside the inclusion

Next, to study the size dependence of the effective thermal conductivity of the resulting composite, the inhomogeneity radius $R$ is set to vary from 1 to $50 \mu \mathrm{m}$ while the inhomogeneity volume fraction is kept constant. More pre-

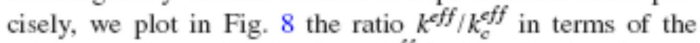
inhomogeneity radius $R$ where $k^{\text {eff }}$ is the effective thermal conductivity calculated by Eqs. (86), (93), (99), and (117) according to the dilute distribution, Mori-Tanaka, self-

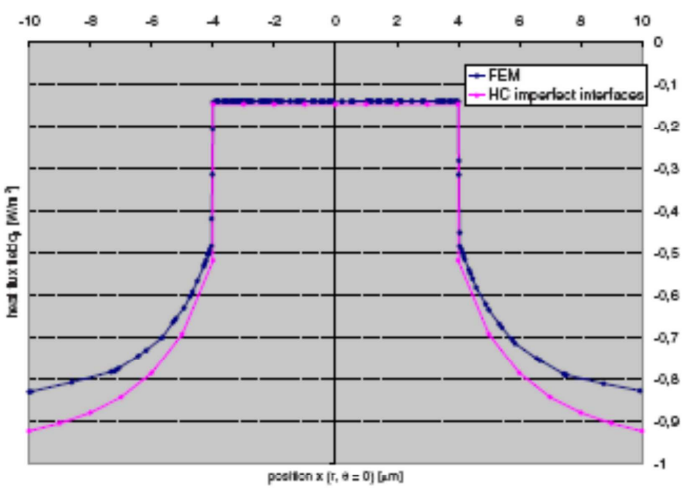

FIG. 6. (Color online) The heat flux fields in the matrix and inclusion obtained by the present analytical method and by FEM for case 1 with inclusion radius $R=4 \mu \mathrm{m}$, thermal conductivity of the interphase $k_{\text {inter }}=20 \mathrm{~W} \mathrm{~m}^{-1} \mathrm{~K}^{-1}$ and thickness of the interphase $h$ $=50 \mathrm{~nm}$. 


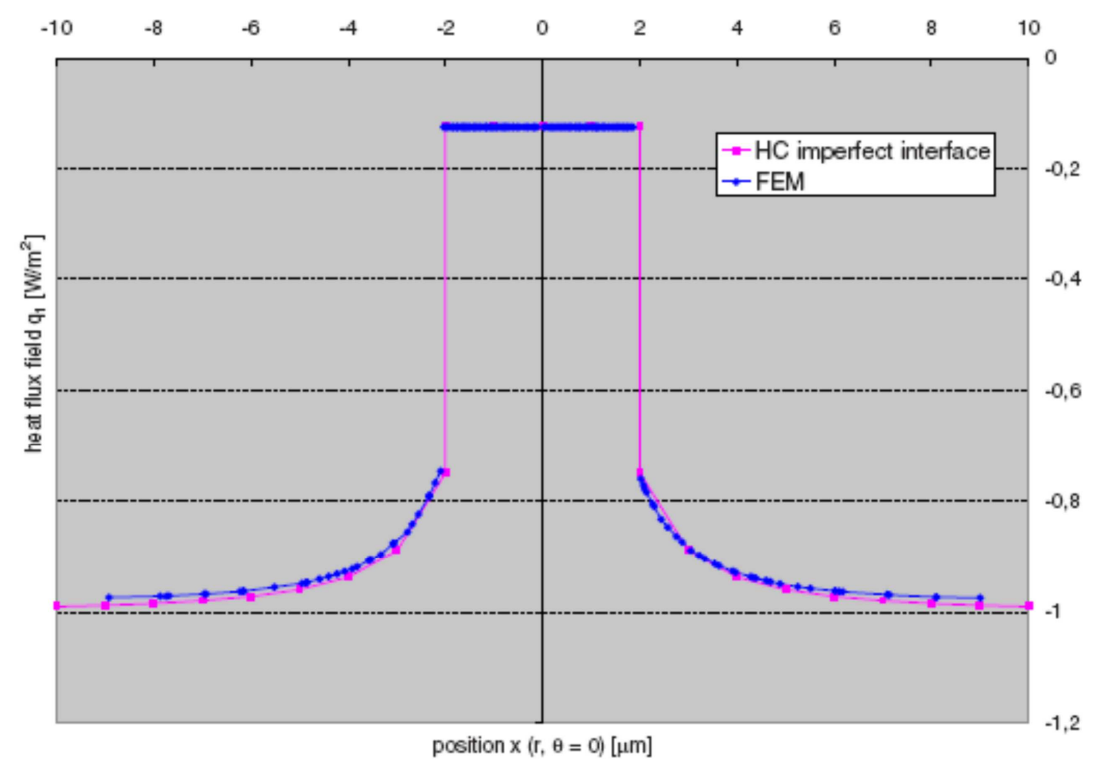

FG. 7. (Color online) The heat flux fields in the matrix and inclusion obtained by the present analytical method and by FEM for case 2 with inclusion radius $R=2 \mu \mathrm{m}$, thermal conductivity of the interphase $k_{\text {inter }}=50 \mathrm{~W} \mathrm{~m}^{-1} \mathrm{~K}^{-1}$ and thickness of the interphase $h=20 \mathrm{~nm}$.

consistent, or generalized self-consistent models, respectively, and $k_{c}^{\text {eff }}$ denotes the effective thermal conductivity without accounting for the surface effects. It is seen from Fig. 8 that (i) $k^{\text {eff }}$ depends on the inhomogeneity radius $R$ while $k_{f f}^{e f f}$ is independent of $R$; (ii) the difference between $k^{e f f}$ and $k_{c}^{\text {fff }}$ decreases when $R$ increases; and (iii) the surface effect becomes negligible when $R$ is larger than $50 \mu \mathrm{m}$.

The ratio $k^{e f f} / k_{c}^{e f f}$ in terms of the inhomogeneity volume fraction $c_{1}$ is presented in Figs. 9 and 10 with two inhomogeneity radii $R=1$ and $20 \mu \mathrm{m}$, respectively. First, Figs. 9 and 10 show that when the inhomogeneity volume fraction increases, the size effect characterized by the ratio $k^{e f f} / k_{c}^{e f f}$ following the dilute distribution, Mori-Tanaka, self-

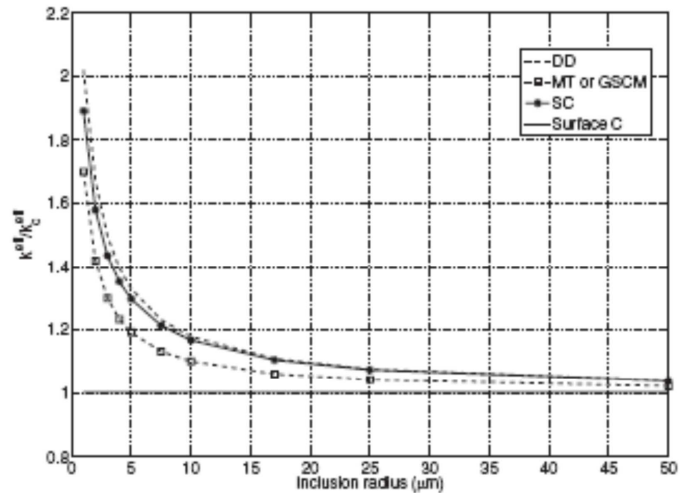

FG. 8. The ratio $k^{\text {eff } / k_{c}^{e f f}}$ versus the inclusion radius $R(\mu \mathrm{m})$ with volume fraction of inclusions $c_{1}=0.3$. consistent, or generalized self-consistent models increases also. Moreover, we see from Figs. 9 and 10 that the surface effect becomes more important as the inhomogeneity radius diminishes at a constant value of $c_{1}$.

\section{CONCLUDING REMARKS}

The interface stress model has widely been used in modeling surface and interfaces effects in nanomaterials and nanostructures. The thermal-conduction counterpart of the interface stress model is the highly conducting interface model. In the context of elasticity, Eshelby's problem for a spherical inclusion embedded in an infinite matrix via an interface characterized by the interface stress model was solved by Sharma and Ganti ${ }^{17}$ and Duan et al. ${ }^{18}$ who showed that Eshelby's strain tensor field inside the spherical inclu-

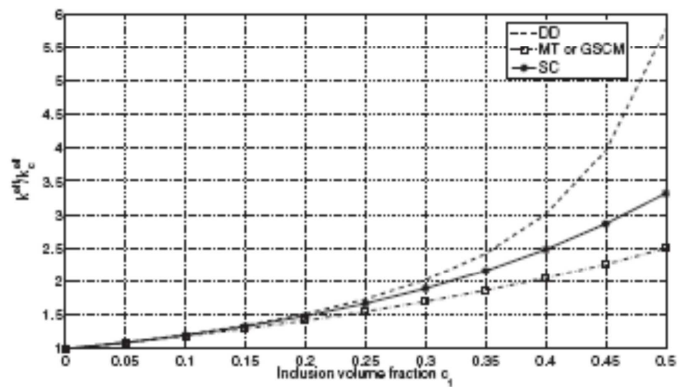

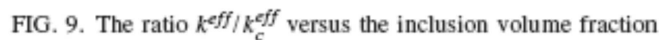
$c_{1}$ with inclusion radius $R=1 \mu \mathrm{m}$. 


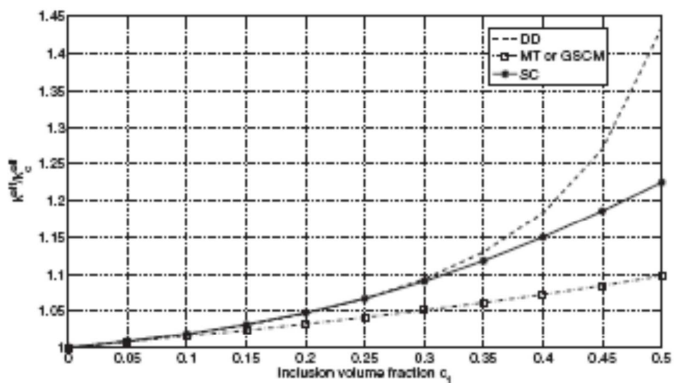

FG. 10. The ratio $k^{e f f} / k_{c}^{e f f}$ versus the inclusion volume fraction $c_{1}$ with inclusion radius $R=20 \mu \mathrm{m}$.

sion is generally no longer uniform. Thus, Eshelby's formalism allowing the transformation of an inhomogeneity problem into an inclusion problem is no more valid. This is because Eshelby's formalism is based on the uniformity of the strain field inside an ellipsoidal inclusion embedded in an infinite matrix and subjected to a prescribed uniform eigenstrain. Unlike the results of Sharma and Ganti ${ }^{17}$ and Duan et $a l^{18}$ for elasticity, the results given by the present paper for thermal conduction show that the temperature gradient field inside a circular or spherical inclusion remains uniform even in the presence of a highly conducting interface. This fact has allowed us to preserve Eshelby's formalism and apply some well-known micromechanical schemes to obtain closed-form expressions for the effective conductivity of composites with highly conducting interfaces. Further, the results derived in the present work hold even when the materials constituting the matrix and inclusions have the most general anisotropy, i.e., orthotropy. This is in contrast with all works reported in the literature on the effective conductivity of composites with imperfect interfaces.

For example, inspired from the elastic inclusion method of Eshelby, Hatta and Taya ${ }^{41}$ studied the steady-state heat conduction in composites made of a matrix in which ellipsoidal inhomogeneities are embedded under the assumption that the materials constituting the matrix and inhomogeneities are isotropic and the interface between them is perfect Later, Hatta and Taya ${ }^{42}$ and Dunn and Taya ${ }^{43}$ extended the work of Hatta and Taya ${ }^{41}$ to composites with coated spheroidal inhomogeneities. As in Hatta and Taya, ${ }^{41}$ the results obtained by Hatta and Taya ${ }^{42}$ and Dunn and Taya ${ }^{43}$ are valid only for isotropic constituents and take the form of infinite series due to the fact that the coated inhomogeneities under consideration are not spherical. These results were then applied to modeling the effects of imperfect interfaces by considering very thin coatings and assimilating them to representing imperfect interfaces. However, in the case where only spherical inhomogeneities are involved and only highly conducting imperfect interfaces are concerned, the method and results presented in the present work have, in comparison with the relevant ones of Hatta and Taya ${ }^{42}$ and Dunn and Taya,$^{43}$ the advantage of being more direct and more explicit in addition to being applicable to anisotropic phases. Indeed, our method consists in directly extending Eshelby's formalism by incorporating highly imperfect interface effects and the parameters characterizing the highly conducting interface model are clearly and explicitly present in the formulas providing estimations of the effective thermal conductivity of composites.

The approach elaborated and results obtained by the present work are straightforwardly transposable to other physically analogous transport phenomena, such as electric conduction, dielectrics, magnetism, diffusion, and flow in porous media. Since a one-to-one correspondence between antiplane elasticity and 2D thermal conduction exists, new results for antiplane anisotropic elasticity can be deduced directly from the $2 \mathrm{D}$ anisotropic results derived above.

\section{APPENDIX: DERIVATION OF Eq. (29)}

First, under the definitions [Eq. (4)] for the projection $\mathbf{P}^{\perp}$ along the unit vector $\mathbf{n}(\mathbf{x})$ and projection $\mathbf{P}$ on the plane tangent to the surface $\Gamma$, let now $\mathbf{w}$ be an arbitrary differentiable vector, we have

$$
\mathbf{w}=\mathbf{w}^{n}+\mathbf{w}^{s}, \quad \mathbf{w}^{n}=\mathbf{P}^{\perp} \mathbf{w}, \quad \mathbf{w}^{s}=\mathbf{P} \mathbf{w},
$$

$$
\nabla \mathbf{w}=\nabla_{n} \mathbf{w}+\nabla_{s} \mathbf{w}, \quad \nabla_{n} \mathbf{w}=\nabla \mathbf{w} \mathbf{P}^{\perp}, \quad \nabla_{s} \mathbf{w}=\nabla \mathbf{w P},
$$

$$
\operatorname{div}_{s} \mathbf{w}=\operatorname{Tr}\left(\nabla_{s} \mathbf{w}\right)=\mathbf{I}^{(d)}:[\nabla \mathbf{w P}] .
$$

Next, due to the fact that the intensity field $\mathbf{e}$ is uniform inside the inhomogeneity $\omega$ and by accounting for the surface thermal behavior corresponding to Eq. (5) and Eqs. (A1)-(A3), we can write

$$
\begin{aligned}
\operatorname{div}_{s} \mathbf{q}^{s} & =k_{s} \operatorname{div}_{s} \mathbf{e}^{s}=k_{s} \operatorname{div}_{s}(\mathbf{P e})=k_{s} \operatorname{Tr}\left[\nabla_{s}(\mathbf{P e})\right] \\
& =k_{s} \mathbf{I}^{(d)}:[\nabla s(\mathbf{P e})] \\
& =k_{s} \mathbf{I}^{(d)}:[\nabla(\mathbf{P e}) \mathbf{P}] \\
& =k_{s} \mathbf{I}^{(d)}:\left\{\nabla\left\{\left[\mathbf{I}^{(d)}-\mathbf{n} \otimes \mathbf{n}\right] \mathbf{e}\right\} \mathbf{P}\right\} \\
& =-k_{s} \mathbf{I}^{(d)}:[(\nabla \mathbf{n} \otimes \mathbf{n}+\mathbf{n} \otimes \nabla \mathbf{n}) \mathbf{e P}] .
\end{aligned}
$$

Moreover, when the inhomogeneity is $3 \mathrm{D}$ spherical or $2 \mathrm{D}$ circular of radius $R$, it is easy to show that

$$
\nabla \mathbf{n}=\frac{1}{R} \mathbf{P}
$$

Substituting Eq. (A5) into Eq. (A4), we have

$$
\begin{aligned}
\operatorname{div}_{s} \mathbf{q}^{s} & =-\frac{k_{s}}{R} \mathbf{I}^{(d)}:[(\mathbf{P} \otimes \mathbf{n}+\mathbf{n} \otimes \mathbf{P}) \mathbf{e P}] \\
& =-\frac{k_{s}}{R}(\mathbf{e} \cdot \mathbf{n}) \mathbf{I}^{(d)}: \mathbf{P}-\frac{k_{s}}{R}(\mathbf{P n}) \cdot(\mathbf{P e}) .
\end{aligned}
$$

Finally, owing to the fact that $\mathbf{I}^{(d)}: \mathbf{P}=\mathbf{I}^{(d)}:\left(\mathbf{I}^{(d)}-\mathbf{n} \otimes \mathbf{n}\right)=d$ -1 and $(\mathrm{Pn}) \cdot(\mathrm{Pe})=\mathbf{0} \cdot \mathbf{e}^{s}=0, \mathrm{Eq} \cdot(\mathrm{A} 6)$ is reduced to

$$
\operatorname{div}_{s} \mathbf{q}^{s}=-\frac{k_{s}}{R}(d-1) \mathbf{e} \cdot \mathbf{n}
$$

This equation is equivalent to Eq. (29). 
*Corresponding author. FAX: $33 \quad$ (0) $160 \quad 957 \quad 799$; hung.lequang@univ-paris-est.fr

${ }^{1}$ Y. Benveniste and T. Miloh, Int. J. Eng. Sci. 24, 1537 (1986).

${ }^{2}$ Y. Benveniste, J. Appl. Phys. 61, 2840 (1987).

${ }^{3}$ D. P. H. Hasselman and L. F. Johnson, J. Compos. Mater. 21, 508 (1987).

${ }^{4}$ R. Lipton and B. Vernescu, Proc. R. Soc. London, Ser. A 452, 329 (1996).

${ }^{5}$ R. Lipton and B. Vernescu, J. Appl. Phys. 79, 8964 (1996).

${ }^{6}$ S. Torquato and M. D. Rintoul, Phys. Rev. Lett. 75, 4067 (1995).

${ }^{7}$ H. Cheng and S. Torquato, Proc. R. Soc. London, Ser. A 453 , 145 (1997).

${ }^{8}$ C.-W. Nan, R. Birringer, D. R. Clarke, and H. Gleiter, J. Appl. Phys. 81, 6692 (1997).

${ }^{9}$ Z. Hashin, J. Appl. Phys. 89, 2261 (2001).

${ }^{10}$ R. Lipton, SIAM J. Appl. Math. 57, 347 (1997).

${ }^{11}$ R. Lipton, J. Mech. Phys. Solids 45, 361 (1997).

${ }^{12}$ T. Miloh and Y. Benveniste, Proc. R. Soc. London, Ser. A 455, 2687 (1999).

${ }^{13} \mathrm{~J}$. Yvonnet, Q.-C. He, and C. Toulemonde, Compos. Sci. Technol. 68, 2818 (2008).

${ }^{14}$ Y. Benveniste, J. Mech. Phys. Solids 54, 708 (2006).

${ }^{15}$ E. Sanchez-Palencia, C. R. Acad. Sci. Paris Ser. A270, 1026 (1970).

${ }^{16}$ H. Pham Huy and E. Sanchez-Palencia, J. Math. Anal. Appl. 47, 284 (1974).

${ }^{17}$ P. Sharma and S. Ganti, J. Appl. Mech. 71, 663 (2004).

${ }^{18}$ H. L. Duan, J. Wang, Z. P. Huang, and B. L. Karihaloo, Proc. R. Soc. London, Ser. A 461, 3335 (2005).

${ }^{19}$ M. E. Gurtin and A. I. Murdoch, Arch. Ration. Mech. Anal. 57, 291 (1975).

${ }^{20}$ T. Chen, M.-S. Chiu, and C.-N. Weng, J. Appl. Phys. 100,
074308 (2006).

${ }^{21}$ J. D. Eshelby, Proc. R. Soc. London, Ser. A 241, 376 (1957).

${ }^{22} \mathrm{H}$. Le Quang, Q.-C. He, and Q.-Z. Zheng, Int. J. Solids Struct. 45, 3845 (2008).

${ }^{23}$ J. D. Eshelby, in Progress in Solid Mechanics, edited by I. N. Sneddon and R. Hill (North-Holland, Amsterdam, 1961), Vol. 2, p. 89.

${ }^{24}$ O. D. Kellogg, Potential Theory (Springer-Verlag, Berlin, 1929), Vol. 39 , p. 1041.

${ }^{25}$ W. D. MacMillan, The Theory of the Potential (Dover, New York, 1958), Vol. 79, p. 8964

${ }^{26}$ N. M. Ferrers, Quart. J. Pure Appl. Math. 14, 1 (1877).

${ }^{27}$ F. W. Dyson, Quart. J. Pure Appl. Math. 25, 259 (1891).

${ }^{28}$ H. G. Beom, Arch. Appl. Mech. 68, 422 (1998).

${ }^{29}$ Z. Hashin, J. Compos. Mater. 2, 284 (1968).

${ }^{30}$ Z. Hashin, J. Appl. Mech. 50, 481 (1983).

${ }^{31}$ C. van der Poel, Rheol. Acta 1, 198 (1958).

${ }^{32}$ J. C. Smith, J. Res. Natl. Bur. Stand. 78A, 355 (1974).

${ }^{33}$ J. C. Smith, J. Res. Natl. Bur. Stand. 79A, 419 (1975).

${ }^{34}$ R. M. Christensen and K. H. Lo, J. Mech. Phys. Solids 27, 315 (1979).

${ }^{35}$ Z. Hashin, NASA Report No. NASA CR-1974, 1972 (unpublished).

${ }^{36}$ E. Herve, Int. J. Solids Struct. 39, 1041 (2002).

${ }^{37}$ Z. Hashin and S. Shtrikman, J. Appl. Phys. 33, 3125 (1962).

${ }^{38}$ H. Le Quang and Q.-C. He, J. Mech. Phys. Solids 55, 1889 (2007).

${ }^{39}$ P. Gilormini, C. R. Acad Sci. Paris Ser. IItb 329, 851 (2001).

${ }^{40}$ Q.-C. He and H. Le Quang, C. R. Mecanique 333, 439 (2005).

${ }^{41} \mathrm{H}$. Hatta and M. Taya, Int. J. Eng. Sci. 24, 1159 (1986).

${ }^{42}$ H. Hatta and M. Taya, J. Appl. Phys. 59, 1851 (1986).

${ }^{43}$ M. L. Dunn and M. Taya, J. Appl. Phys. 73, 1711 (1993). 